\title{
STABILITY AND CONVERGENCE OF A FINITE ELEMENT METHOD FOR REACTIVE TRANSPORT IN GROUND WATER
}

\author{
Zhangxin Chen and Richard E. Ewing*
}

\begin{abstract}
An explicit finite element method is used to solve the linear convection-diffusionreaction equations governing contaminant transport in ground water flowing through an adsorbing porous medium. The use of discontinuous finite elements for the convective part of the equations combined with mixed finite elements for the diffusive part renders the method for the concentration solution, which displays strong gradients, trivially conservative and fully parallelizable. We carry out a stability and convergence analysis. In particular, the method is proven to satisfy a maximum principle, to be total variation bounded, and to converge to the unique weak solution of the equations. Special attention is paid to the convective part of the equations. Numerical simulations are presented and discussed.
\end{abstract}

Key words. convection-diffusion-reaction equation, finite element and volume method, conservation law, stability, convergence, mixed method

AMS subject classifications. 65N30, 65N10, 76S05, 76T05

1. Introduction. In this paper we propose and analyze a finite element method for solving the linear convection-diffusion-reaction equation:

$$
\frac{\partial}{\partial t}(\Phi u)+\operatorname{div}(V u-D \nabla u)=-K u
$$

which describes the transport of a solute in a fluid phase flowing through a porous medium [1], [16]. In this case, $u=u(t, x, y)$ is the concentration of the solute in the fluid phase for which we solve (1.1), $V=V(t, x, y)$ is the Darcy velocity of fluid, $\Phi$ is the volume fraction-dependent constant, $D$ is the diffusion constant, and $K=K(t, x, y) \geq 0$ is the first-order chemical reaction rate. This equation, while formally parabolic, is more nearly hyperbolic in practice [4]. In recent years many finite element methods have been proposed to solve this important partial differential equation. The classes of optimal spatial methods and characteristic methods have been extensively studied [2], [9], [15], [17], [18], for example. However, all these finite element methods are defined by taking advantage of the parabolicity of the equation for the concentration $u$. As a result, the solution of the differential equation is required very smooth in the derivation of error estimates, and the constants for the error estimates blow up as the coefficient of the diffusion term goes to zero.

In this paper we propose and analyze a finite element method for numerically solving (1.1). It is similar to a finite element method introduced in [5], [3], [6], [10], [11] in that we approximate the convective part of the equation using a upwinding discontinuous finite element method or a upwinding finite volume method [20], [19]. We use, however, a mixed finite element method for the diffusive part of (1.1) [8]. The main advantages of this method are that it is trivially conservative and fully parallelizable, and that it can capture discontinuities within a couple of elements without producing spurious oscillations.

\footnotetext{
* Department of Mathematics and the Institute for Scientific Computation, Texas A\&M University, College Station, TX 77843. Current address of Z. Chen: Department of Mathematics, Box 156, Dedman College, Southern Methodist University, Dallas, Texas 75275-0156. Partly supported by the Department of Energy under contract DE-ACOS-840R21400. email: zchen@golem.math.smu.edu, ewing@ewing.tamu.edu.
} 
A stability and convergence analysis is carried out here for the finite element method for equation (1.1) in two space dimensions. While a stability analysis was completed for the similar approach for the two-dimensional semiconductor device equations in [6], we are here able to prove much stronger results than those obtained in [6]. Namely, besides a strong maximum principle, the boundedness of the total variation and the modulus of continuity in time of the approximate solution is proven here; only an estimate on the weak derivatives of the approximate solution is given in [6]. These properties suffice to show that the numerical method converges to the weak solution of the differential equation; in [6], however, convergence of the approximate solutions to the weak solution is proven under the assumption that there is a convergent subsequence. It is also emphasized that this paper contains the first stability analysis for the two-dimensional equation (1.1) with the diffusion term included and the first convergence analysis for (1.1) with the boundary conditions. The properties derived in this paper will be exploited in a forthcoming paper where error estimates will be obtained with minimum requirements on the solution and with the property that the constant for the error estimates does not involve the small diffusion coefficient. Especially, the error estimates apply to the case of $D$ equal to zero.

The equation (1.1) is completed by specifying the boundary and initial conditions:

$$
\begin{aligned}
& \partial u / \partial \nu=0, \quad(x, y) \in \partial \Omega_{1}, \quad t \in J, \\
& u=u_{D}, \quad(x, y) \in \partial \Omega_{2}, \quad t \in J, \\
& u(0, x, y)=u_{\text {init }}(x, y), \quad(x, y) \in \Omega,
\end{aligned}
$$

where $J=(0, T), \Omega=(0,1)^{2}, \partial \Omega=\partial \Omega_{1} \cup \partial \Omega_{2}$ with $\partial \Omega_{1} \cap \partial \Omega_{2}=\emptyset$ and $\Omega_{1}$ containing the endpoints of its segments, and $\nu$ denotes the normal unit-vector to $\partial \Omega$. The boundary conditions need to be modified properly in the case of $D=0$. Namely, only the inflow boundary condition is imposed for the concentration (see (3.9) below). Moreover, in this case, note that, while the equation (1.1) is analogous to a classical conservation law, the value of the Darcy velocity $V$ at a point $(t, x, y)$ contains the information of all the values of the solution $u(t, \cdot, \cdot)$ on $\Omega$. Hence a perturbation of the solution $u$ at any given point of the domain has a global effect immediately. This is in sharp contrast with the classical conservation laws where local perturbations of the solution have a local effect in finite time.

The rest of the paper is organized as follows. The finite element method is defined in the next section. Then, in $\S 3$ we state and discuss our main results on a maximum principle (Theorem 3.1), a total variation boundedness of the scheme (Theorem 3.2), continuity with respect to data (Theorem 3.3), and convergence to the weak solution (Theorem 3.4). The proofs of these properties are carried out in $\S 4, \S 5$, $\S 6$, and $\S 7$, respectively. Numerical results are displayed in $\S 8$. These numerical results are devised to test the performance of the method and to indicate the order of convergence. Finally, a concluding remark is given in $\S 9$.

2. The finite element method. In this section we define the finite element method for approximating the solution of the differential system (1.1). Toward that end, let $\left\{x_{i+1 / 2}\right\}_{i=0}^{n_{x}} \times\left\{y_{j+1 / 2}\right\}_{j=0}^{n_{y}}$ be a partition of $\Omega$ with $x_{1 / 2}=y_{1 / 2}=0$ and $x_{n_{x}+1 / 2}=y_{n_{y}+1 / 2}=1$ and let $\left\{t^{n}\right\}_{n=0}^{n_{T}}$ be a partition of $[0, T]$ with $t^{0}=0$ and $t^{n_{T}}=T$. Then, we introduce the following notation

$$
x_{i}=\left(x_{i-1 / 2}+x_{i+1 / 2}\right) / 2, \quad y_{j}=\left(y_{j-1 / 2}+y_{j+1 / 2}\right) / 2,
$$




$$
\begin{array}{ll}
I_{i}^{x}=\left(x_{i-1 / 2}, x_{i+1 / 2}\right), & I_{j}^{y}=\left(y_{j-1 / 2}, y_{j+1 / 2}\right), \\
\Delta x_{i}=x_{i+1 / 2}-x_{i-1 / 2}, & \Delta y_{j}=y_{j+1 / 2}-y_{j-1 / 2}, \\
J^{n}=\left[t^{n}, t^{n+1}\right), & \Delta t^{n}=t^{n+1}-t^{n}, \\
\Delta x=\max _{1 \leq i \leq n_{x}} \Delta x_{i}, & \Delta y=\max _{1 \leq i \leq n_{y}} \Delta y_{i}, \\
\Delta t=\max _{0 \leq n \leq n_{T}} \Delta t^{n}, & h=\max \{\Delta x, \Delta y\} .
\end{array}
$$

We tacitly assume that each exterior edge has imposed on it either Dirichlet or Neumann conditions, but not both. Associated with these partitions, we introduce the spaces

$$
\begin{gathered}
Q_{h}=\left\{v \in H(\operatorname{div} ; \Omega):\left.v\right|_{I_{i}^{x} \times I_{j}^{y}}=\left(a_{i, j}^{1}+a_{i, j}^{2} x, a_{i, j}^{3}+a_{i, j}^{4} y\right), a_{i, j}^{k} \in \mathbb{R},\right. \\
\left.i=1, \cdots, n_{x}, j=1, \cdots, n_{y},\left.\quad v \cdot \nu\right|_{\partial \Omega_{1}}=0\right\}, \\
W_{h}=\left\{w \in L^{\infty}(\Omega):\left.w\right|_{I_{i}^{x} \times I_{j}^{y}} \in P^{0}\left(I_{i}^{x} \times I_{j}^{y}\right), \quad i=1, \cdots, n_{x}, j=1, \cdots, n_{y}\right\}, \\
W_{\Delta t}=\left\{w \text { right continuous : }\left.w\right|_{J^{n}} \in P^{0}\left(J^{n}\right), n=0, \cdots, n_{T}-1\right\} .
\end{gathered}
$$

If $v \in Q_{h}, v_{i+1 / 2, j}$ and $v_{i, j+1 / 2}$ denote $v\left(x_{i+1 / 2}, y_{j}\right)$ and $v\left(x_{i}, y_{j+1 / 2}\right)$, respectively. If $w \in W_{h}$, then $w_{i, j}$ represents the constant value $w(x, y),(x, y) \in I_{i}^{x} \times I_{j}^{y} . w^{n}$ indicates the constant $w(t), t \in J^{n}$, if $w \in W_{\Delta t}$. For notational and expositional convenience, let $\Delta x_{0}=\Delta x_{1}, \Delta x_{n_{x}+1}=\Delta x_{n_{x}}, \Delta y_{0}=\Delta y_{1}, \Delta y_{n_{y}+1}=\Delta y_{n_{y}}, \Delta x_{i+1 / 2}=\left(\Delta x_{i}+\right.$ $\left.\Delta x_{i+1}\right) / 2, i=1, \cdots, n_{x}, \Delta y_{j+1 / 2}=\left(\Delta y_{j}+\Delta y_{j+1}\right) / 2, j=1, \cdots, n_{y}$, and $\Phi=1$. Finally, define the notation $v^{+}=\max \{v, 0\}$ and $v^{-}=\min \{v, 0\}$.

Let $P_{Q_{h}}, P_{W_{h}}$, and $P_{W_{\Delta t}}$ denote the $L^{2}$-projections into $Q_{h}, W_{h}$, and $W_{\Delta t}$, respectively. To discretize (1.1), we first discretize the data as follows:

$$
\begin{aligned}
u_{\text {init }, h} & =P_{W_{h}} u_{\text {init }}, \\
u_{D, \Delta t} & =P_{W_{\Delta t}} u_{D}, \\
V_{h} & =P_{Q_{h}} V .
\end{aligned}
$$

The subscript $h$ is omitted below when no ambiguity occurs. Then the approximate solution $u_{h} \in W_{\Delta t} \otimes W_{h}$ is required to satisfy the equation, for $n=0, \cdots, n_{T}-1, i=$ $1, \cdots, n_{x}$, and $j=1, \cdots, n_{y}$ :

$$
\begin{aligned}
& \frac{u_{i, j}^{n+1}-u_{i, j}^{n}}{\Delta t^{n}}+\frac{f_{1, i+1 / 2, j}^{n}-f_{1, i-1 / 2, j}^{n}}{\Delta x_{i}}+\frac{f_{2, i, j+1 / 2}^{n}-f_{2, i, j-1 / 2}^{n}}{\Delta y_{j}} \\
& -\frac{D}{\Delta x_{i}}\left(q_{1, i+1 / 2, j}^{n}-q_{1, i-1 / 2, j}^{n}\right)-\frac{D}{\Delta y_{j}}\left(q_{2, i, j+1 / 2}^{n}-q_{2, i, j-1 / 2}^{n}\right)=-K_{i, j}^{n} u_{i, j}^{n},
\end{aligned}
$$

where

$$
\begin{aligned}
& f_{1, i-1 / 2, j}^{n}=u_{i-1, j}^{n} V_{1, i-1 / 2, j}^{n^{+}}+u_{i, j}^{n} V_{1, i-1 / 2, j}^{n^{-}}, \\
& f_{2, i, j-1 / 2}^{n}=u_{i, j-1}^{n} V_{2, i, j-1 / 2}^{n^{+}}+u_{i, j}^{n} V_{2, i, j-1 / 2}^{n^{-}},
\end{aligned}
$$

and the function $q_{h}=\left(q_{1}, q_{2}\right) \in W_{\Delta t} \otimes Q_{h}$ is the solution of

$$
\left(q_{h}\left(t^{n}\right), v_{h}\right)=-\left(u_{h}\left(t^{n}\right), \operatorname{div} v_{h}\right)+\left\langle u_{D, \Delta t}, v_{h} \cdot \nu\right\rangle_{\partial \Omega_{D}}, \quad \forall v_{h} \in Q_{h} .
$$

After the mass matrix has been mass-lumped [22], the expression for the degrees of 
freedom of $q_{h}$ is taken as follows:

$$
\begin{aligned}
& q_{1, i-1 / 2, j}^{n}=\left(u_{i, j}^{n}-u_{i-1, j}^{n}\right) / \Delta x_{i-1 / 2}, \\
& q_{2, i, j-1 / 2}^{n}=\left(u_{i, j}^{n}-u_{i, j-1}^{n}\right) / \Delta y_{j-1 / 2} .
\end{aligned}
$$

Finally, the Neumann boundary condition (1.2a) is discretized by the usual reflection principle, and on $\partial \Omega_{2} u_{h}$ is defined by $u_{D, \Delta t}$. This implies that, if $\left(x_{1 / 2}, y_{j}\right)$ lies on the Neumann boundary $\partial \Omega_{1}, u_{0, j}^{n}$ in (2.2) and the subsequent analysis is calculated by

$$
u_{0, j}^{n}=u_{1, j}^{n}
$$

if it is on the Dirichlet boundary $\partial \Omega_{2}, u_{0, j}^{n}$ is computed by

$$
u_{0, j}^{n}=u_{D, \Delta t}^{n}\left(x_{1 / 2}, y_{j}\right) .
$$

Similar extensions hold for $u_{i, 0}^{n}, u_{n_{x}+1, j}^{n}$, and $u_{i, n_{y}+1}^{n}$ in (2.2) and the subsequent analysis.

Note that the lowest-order Raviart-Thomas mixed method [21] over rectangles has been used in (2.2a). Since the elements in $Q_{h}$ have continuous normal components on interelement edges, the numerical fluxes $f_{1, i-1 / 2, j}^{n}$ and $f_{2, i, j-1 / 2}^{n}$ in $(2.2 \mathrm{~b})$ and (2.2c) are well defined. Furthermore, if appropriate approximations of the coefficient $V_{h}$ are introduced and the mass-lumping technique is used as in (2.2d) and (2.2e), the conservative scheme $(2.2 \mathrm{a})$ can be deduced from the discontinuous finite element method [7], [12] or from the finite volume method [20], [19] combined with the mixed finite element method [22]. Finally, the scheme applies to the case of $D=0$.

The following approximation properties are used later [14], [21]:

$$
\begin{aligned}
& \left\|V_{h}^{n}\right\|_{L^{\infty}(\Omega)} \leq C_{0}\left\|V^{n}\right\|_{L^{\infty}(\Omega)}, \\
& \left\|\operatorname{div} V_{h}^{n}\right\|_{B V(\Omega)} \leq C_{0}\left\|\operatorname{div} V^{n}\right\|_{B V(\Omega)},
\end{aligned}
$$

for each $n$. Moreover, since the operator $P_{Q_{h}}$ is locally defined, we have for each $n$ :

$$
\left\|V^{n}-V_{h}^{n}\right\|_{L^{\infty}\left(I_{i}^{x} \times I_{j}^{y}\right)} \leq C_{0}\left(\Delta x_{i}+\Delta y_{j}\right)|| \nabla V^{n} \|_{L^{\infty}\left(I_{i}^{x} \times I_{j}^{y}\right)},
$$

for $i=1, \ldots, n_{x}, j=1, \ldots, n_{y}$, where $C_{0}$ is independent of $i$ and $j$.

3. Stability and convergence results. In this section we state and discuss the stability and convergence results of the scheme (2.2). Let $Q_{T}=T \times \Omega$. We assume that the data satisfy the following conditions:

$$
\begin{aligned}
& u_{\text {init }}, u_{D} \in\left[0, u^{\star}\right], \\
& V \in\left(L^{\infty}\left(Q_{T}\right)\right)^{2}, \nabla V \in\left(L^{\infty}\left(Q_{T}\right)\right)^{4}, \\
& u_{D} \in L^{\infty}\left(J ; B V\left(\partial \Omega_{2}\right)\right), \\
& u_{D} \in L^{1}\left(\partial \Omega_{2} ; B V(J)\right), \\
& \operatorname{div} V \in L^{\infty}(J ; B V(\Omega)), \\
& K \in\left[0, K^{\star}\right], \\
& u_{\text {init }} \in B V(\Omega), K \in L^{\infty}(J ; B V(\Omega)) .
\end{aligned}
$$

For expositional convenience, let

$$
V_{1}^{\star}=C_{0}\left\|V_{1}\right\|_{L^{\infty}\left(Q_{T}\right)}, \quad V_{2}^{\star}=C_{0}\left\|V_{2}\right\|_{L^{\infty}\left(Q_{T}\right)}, \quad V_{D}^{\star}=C_{0}\|\operatorname{div} V\|_{L^{\infty}\left(Q_{T}\right)} .
$$


Theorem 3.1 (Stability). Suppose that (3.1a), (3.1b), (3.1f), and for $n=$ $0, \cdots, n_{T}-1$ the following Courant-Friedrichs-Lewy (CFL) condition are satisfied:

$$
\Delta t^{n} \leq \frac{1}{D_{i j}^{\star}+2 V_{1}^{\star} / \Delta x_{i}+2 V_{2}^{\star} / \Delta y_{j}}, \quad i=1, \cdots, n_{x}, j=1, \cdots, n_{y},
$$

where $D_{i j}^{\star}=\frac{D}{\Delta x_{i}}\left(\frac{1}{\Delta x_{i+1 / 2}}+\frac{1}{\Delta x_{i-1 / 2}}\right)+\frac{D}{\Delta y_{j}}\left(\frac{1}{\Delta y_{j+1 / 2}}+\frac{1}{\Delta y_{j-1 / 2}}\right)+K^{\star}$. Then

$$
0 \leq u_{h}(t, x, y) \leq e^{t V_{D}^{\star}} u^{\star}, \quad(t, x, y) \in Q_{T} .
$$

In addition, if

$$
\left(\operatorname{div} V_{h}^{n}\right)_{i j}+K_{i, j}^{n} \geq 0
$$

then we have

$$
0 \leq u_{h}(t, x, y) \leq u^{\star}, \quad(t, x, y) \in Q_{T} .
$$

Obviously, since $K \geq 0,(3.4)$ is satisfied if $V_{1}$ is nondecreasing in $x$ and $V_{2}$ is nondecreasing in $y$, or $\operatorname{div} V$ is uniformly positive by the definition of $P_{Q_{h}}$ [21].

Define, for $n=0, \cdots, n_{T}$,

$$
\|\left. u_{h}^{n}\right|_{B V(\Omega)}=\sum_{i=0}^{n_{x}} \sum_{j=0}^{n_{y}}\left(\left|u_{i+1, j}^{n}-u_{i, j}^{n}\right| \Delta y_{j}+\left|u_{i, j+1}^{n}-u_{i, j}^{n}\right| \Delta x_{i}\right),
$$

and set

$$
\Delta x_{\star}=\min \left\{\Delta x_{i}, i=1, \cdots, n_{x}\right\}, \quad \Delta y_{\star}=\min \left\{\Delta y_{j}, j=1, \cdots, n_{y}\right\} .
$$

Theorem 3.2 (TVB). Assume that (3.1) and for $n=0, \cdots, n_{T}-1$ the following CFL condition are satisfied:

$$
\Delta t^{n} \leq \frac{1}{D_{i j}^{\star}+3 V_{1}^{\star} / \Delta x_{\star}+3 V_{2}^{\star} / \Delta y_{\star}}, \quad i=1, \cdots, n_{x}, j=1, \cdots, n_{y} .
$$

Then there is a constant $C_{1}$ depending solely on the data and $T$ such that

$$
\begin{aligned}
\left\|u_{h}\right\|_{L^{\infty}(J ; B V(\Omega))} \leq C_{1}\{ & 1+\|K\|_{L^{\infty}(J ; B V(\Omega))} \\
& \left.+D\left(\frac{1}{\Delta x_{\star}}+\frac{1}{\Delta y_{\star}}\right)\left\|u_{D}\right\|_{L^{\infty}\left(J ; B V\left(\partial \Omega_{2}\right)\right)}\right\} .
\end{aligned}
$$

We remark that either in the case of $D=0$ or in the case of $u_{D}$ being constant in space, (3.7) shows that the total variation of the solution $u_{h}$ is bounded. The latter case means that the total variation of the solution $u_{h}$ in the one-dimensional case is always bounded since $u_{D}$ is constant in this case. The numerical experiments given in $\S 8$ show that the bounds in (3.7) and (3.8) below are sharp when $D \neq 0$ and $\left\|u_{D}\right\|_{L^{\infty}\left(J ; B V\left(\partial \Omega_{2}\right)\right)} \neq 0$, in the sense that the left-hand sides of the inequalities (3.7) and (3.8) blow up as $\Delta x_{*}$ or $\Delta y_{*}$ converges to zero (see Example 4 in $\S 8$ ).

In the following, $v_{h}$ stands for the approximate solution of (1.1) and (1.2) with the data $v_{\text {init }}$ and $v_{D}$ satisfying the conditions (3.1a), (3.1c), and (3.1d). 
Theorem 3.3 (Continuity with Respect to Data). Assume that the hypotheses of Theorem 3.1 are satisfied for both sets of data. Then there exists a constant $C_{2}$ depending only on the data and $T$ such that

$$
\begin{aligned}
\left\|u_{h}-v_{h}\right\|_{L^{\infty}\left(J ; L^{1}(\Omega)\right)} \leq C_{2}\{ & \left(1+D\left(\frac{1}{\Delta x_{\star}}+\frac{1}{\Delta y_{\star}}\right)\right) \\
& \left.\times\left\|u_{D}-v_{D}\right\|_{L^{\infty}\left(J ; L^{1}\left(\partial \Omega_{2}\right)\right)}+\left\|u_{\text {init }}-v_{\text {init }}\right\|_{L^{1}(\Omega)}\right\} .
\end{aligned}
$$

As for the convergence result, we now consider a simple case where $D=0$. In this case Theorem 3.2 implies the total variation boundedness of the numerical scheme as remarked above, which together with Theorem 3.3 yields the following convergence result (see $\S 7$ ). For nonzero $D$, concrete error estimates for the numerical scheme (2.2) will be obtained in the work mentioned earlier.

In the simple case where $D=0$ the boundary conditions (1.2a) and (1.2b) are replaced by the following inflow boundary condition:

$$
u=u_{D}, \quad(x, y) \in \partial \Omega_{-}, \quad t \in J,
$$

where $\partial \Omega_{-}=\{(x, y) \in \partial \Omega:(V \cdot \nu)(x, y)<0\}$. We now extend the numerical flux introduced in $(2.2)$ to the general setting:

$$
f\left(u_{\text {left }}, u_{\text {right }} ; \alpha\right)=u_{\text {left }} \alpha^{+}+u_{\text {right }} \alpha^{-} .
$$

Also, we define

$$
\mathcal{C}_{0}^{1}([0, T) \times \bar{\Omega})=\left\{\varphi \in \mathcal{C}^{1}(\bar{J} \times \bar{\Omega}): \varphi(T, x, y)=0,(x, y) \in \bar{\Omega}\right\} .
$$

Then a weak solution of the differential equation given by (1.1) with $D=0,(3.9)$, and (1.2c) is defined to be a function $u \in L^{\infty}(J ; B V(\Omega))$ satisfying the weak formulation:

$$
\begin{aligned}
& \left(u, \varphi_{t}\right)_{Q_{T}}+(u V, \nabla \varphi)_{Q_{T}}+\left(u_{\text {init }}, \varphi\right)_{\{t=0\} \times \Omega} \\
& \quad-\left(f\left(u, u_{D} ; V \cdot \nu\right), \varphi\right)_{J \times(\partial \Omega)}-(K u, \varphi)_{Q_{T}}=0, \quad \forall \varphi \in \mathcal{C}_{0}^{1}([0, T) \times \bar{\Omega}),
\end{aligned}
$$

where $(\cdot, \cdot)_{S}$ denotes the inner product in $L^{2}(S)$ for some set $S$. Note that the role of the flux $f$ is to select the correct boundary value for $u$, and that the smoothness hypothesis on $V$ guarantees the uniqueness of weak solution to (3.10).

Theorem 3.4 (Convergence). Assume that the hypotheses of Theorem 3.2 are satisfied. Then the sequence $\left\{u_{h}\right\}_{h>0}$ produced by the scheme (2.2) converges in $L^{\infty}\left(J ; L^{1}(\Omega)\right)$ to the unique solution of (3.10). Moreover, $u \in L^{\infty}(J ; B V(\Omega))$.

4. Proof of the maximum principle. In this section we prove Theorem 3.1. Let

$$
U^{n}=\max \left\{u_{i, j}^{n}, 0 \leq i \leq n_{x}+1,0 \leq j \leq n_{y}+1\right\} .
$$

Lemma 4.1. Suppose that

$$
\begin{aligned}
1 & -\frac{\Delta t^{n}}{\Delta x_{i}}\left(V_{1, i+1 / 2, j}^{n^{+}}-V_{1, i-1 / 2, j}^{n^{-}}\right)-\frac{\Delta t^{n}}{\Delta y_{j}}\left(V_{2, i, j+1 / 2}^{n^{+}}-V_{2, i, j-1 / 2}^{n^{-}}\right) \\
- & \frac{D \Delta t^{n}}{\Delta x_{i}}\left(\frac{1}{\Delta x_{i+1 / 2}}+\frac{1}{\Delta x_{i-1 / 2}}\right)-\frac{D \Delta t^{n}}{\Delta y_{j}}\left(\frac{1}{\Delta y_{j+1 / 2}}+\frac{1}{\Delta y_{j-1 / 2}}\right)-K_{i, j}^{n} \Delta t^{n} \geq 0 .
\end{aligned}
$$

Then, if

$$
0 \leq u_{i, j}^{n}, \quad 0 \leq i \leq n_{x}+1,0 \leq j \leq n_{y}+1
$$


we have, for $0 \leq i \leq n_{x}+1$ and $0 \leq j \leq n_{y}+1$,

$$
0 \leq u_{i, j}^{n+1} \leq U^{n+1} \leq\left\{1+\Delta t^{n} \max _{I_{i}^{x} \times I_{j}^{y}}\left\{\left|\operatorname{div} V_{h}^{n}\right|\right\}\right\} U^{n} .
$$

In addition, if

$$
\left(\operatorname{div} V_{h}^{n}\right)_{i j}+K_{i, j}^{n} \geq 0
$$

we have

$$
0 \leq u_{i, j}^{n+1} \leq U^{n}, \quad 0 \leq i \leq n_{x}+1,0 \leq j \leq n_{y}+1 .
$$

Proof. For $i=1, \cdots, n_{x}$ and $j=1, \cdots, n_{y}$, it follows from (2.2) that

$$
u_{i, j}^{n+1}=A_{i+1, j}^{n} u_{i+1, j}^{n}+A_{i, j+1}^{n} u_{i, j+1}^{n}+B_{i, j}^{n} u_{i, j}^{n}+E_{i-1, j}^{n} u_{i-1, j}^{n}+E_{i, j-1}^{n} u_{i, j-1}^{n},
$$

where

$$
\begin{aligned}
& A_{i+1, j}^{n}=-\frac{\Delta t^{n}}{\Delta x_{i}} V_{1, i+1 / 2, j}^{n^{-}}+\frac{D \Delta t^{n}}{\Delta x_{i} \Delta x_{i+1 / 2}}, \\
& A_{i, j+1}^{n}=-\frac{\Delta t^{n}}{\Delta y_{j}} V_{2, i, j+1 / 2}^{n^{-}}+\frac{D \Delta t^{n}}{\Delta y_{j} \Delta y_{j+1 / 2}}, \\
& B_{i, j}^{n}=1-\frac{\Delta t^{n}}{\Delta x_{i}}\left(V_{1, i+1 / 2, j}^{n^{+}}-V_{1, i-1 / 2, j}^{n^{-}}\right)-\frac{\Delta t^{n}}{\Delta y_{j}}\left(V_{2, i, j+1 / 2}^{n^{+}}-V_{2, i, j-1 / 2}^{n^{-}}\right) \\
& \quad-\frac{D \Delta t^{n}}{\Delta x_{i}}\left(\frac{1}{\Delta x_{i+1 / 2}}+\frac{1}{\Delta x_{i-1 / 2}}\right)-\frac{D \Delta t^{n}}{\Delta y_{j}}\left(\frac{1}{\Delta y_{j+1 / 2}}+\frac{1}{\Delta y_{j-1 / 2}}\right)-K_{i, j}^{n} \Delta t^{n}, \\
& E_{i-1, j}^{n}=\frac{\Delta t^{n}}{\Delta x_{i}} V_{1, i-1 / 2, j}^{n^{+}}+\frac{D \Delta t^{n}}{\Delta x_{i} \Delta x_{i-1 / 2}}, \\
& E_{i, j-1}^{n}=\frac{\Delta t^{n}}{\Delta y_{j}} V_{2, i, j-1 / 2}^{n^{+}}+\frac{D \Delta t^{n}}{\Delta y_{j} \Delta y_{j-1 / 2}} .
\end{aligned}
$$

Then, by (4.1), we see that

$$
A_{i+1, j}^{n}, A_{i, j+1}^{n}, B_{i, j}^{n}, E_{i-1, j}^{n}, E_{i, j-1}^{n} \geq 0,
$$

so that, by (4.2),

$$
u_{i, j}^{n+1} \geq 0, \quad i=1, \cdots, n_{x}, j=1, \cdots, n_{y} .
$$

Furthermore, by the definition of $Q_{h}$ and (4.2),

$$
\begin{aligned}
u_{i, j}^{n+1} \leq(1- & \frac{\Delta t^{n}}{\Delta x_{i}}\left(V_{1, i+1 / 2, j}^{n}-V_{1, i-1 / 2, j}^{n}\right) \\
& \left.-\frac{\Delta t^{n}}{\Delta y_{j}}\left(V_{2, i, j+1 / 2}^{n}-V_{2, i, j-1 / 2}^{n}\right)-K_{i, j}^{n} \Delta t^{n}\right) U^{n} \\
= & \left(1-\Delta t^{n}\left(\operatorname{div} V_{h}^{n}\right)_{i, j}-K_{i, j}^{n} \Delta t^{n}\right) U^{n},
\end{aligned}
$$

which implies (4.3) immediately since $K \geq 0$, and together with (4.4) yields (4.5).

Lemma 4.2. If for $i=1, \cdots, n_{x}$ and $j=1, \cdots, n_{y}$

$$
\Delta t^{n} \leq \frac{1}{D_{i j}^{\star}+2|| V_{h 1}^{n}\left\|_{L^{\infty}(\Omega)} / \Delta x_{i}+2|| V_{h 2}^{n}\right\|_{L^{\infty}(\Omega)} / \Delta y_{j}},
$$

where $D_{i j}^{\star}$ is defined as in Theorem 3.1, then (4.1) is satisfied.

The lemma follows obviously from the inequality (4.1) and the definition of $D_{i j}^{\star}$. 
We are now ready to prove Theorem 3.1 by means of induction on $n$.

Proof of Theorem 3.1. For $n=0$, the results (3.3) and (3.5) follow trivially from the assumption (3.1a). Let the results be true up to $n$. By Lemma 4.2 and (2.3a), (3.2) clearly implies (4.1). Then iterating (4.3) on $n$ and using the induction hypothesis and $(2.3 \mathrm{~b})$ yield that

$$
0 \leq u_{i, j}^{n+1} \leq \mathrm{e}^{t^{n+1}} V_{D}^{\star} U^{0}, i=1, \cdots, n_{x}, j=1, \cdots, n_{y} .
$$

Consequently, by (3.1a), (3.3) follows.

If (3.4) is true, so is (4.4). Then, in this case, it follows from (4.5) and the induction hypothesis that

$$
0 \leq u_{i, j}^{n+1} \leq U^{n} \leq U^{0}, i=1, \cdots, n_{x}, j=1, \cdots, n_{y},
$$

which implies (3.5) by (3.1a).

5. Proof of total variation boundedness. In this section we prove Theorem 3.2. In order to fix ideas, let

$$
\begin{aligned}
& \partial \Omega_{2}=\{(x, y): x=0,0<y<1\} \cup\{(x, y): x=1,0<y<1\}, \\
& \partial \Omega_{1}=\partial \Omega \backslash \partial \Omega_{2}
\end{aligned}
$$

other cases can be treated similarly.

LEMmA 5.1. For $i=0$ and $j=1, \cdots, n_{y}$,

$$
\begin{aligned}
u_{1, j}^{n+1}-u_{0, j}^{n+1}= & u_{0, j}^{n}-u_{0, j}^{n+1}+\left(-\frac{\Delta t^{n}}{\Delta x_{1}} V_{1,3 / 2, j}^{n^{-}}+\frac{D \Delta t^{n}}{\Delta x_{3 / 2} \Delta x_{1}}\right)\left(u_{2, j}^{n}-u_{1, j}^{n}\right) \\
& +\left(-\frac{\Delta t^{n}}{\Delta y_{j}} V_{2,1, j+1 / 2}^{n^{-}}+\frac{D \Delta t^{n}}{\Delta y_{j+1 / 2} \Delta y_{j}}\right)\left(u_{1, j+1}^{n}-u_{0, j+1}^{n}\right) \\
& +\left\{1-\frac{\Delta t^{n}}{\Delta x_{1}} V_{1,1 / 2, j}^{n^{+}}+\frac{\Delta t^{n}}{\Delta y_{j}} V_{2,1, j+1 / 2}^{n^{-}}-\frac{\Delta t^{n}}{\Delta y_{j}} V_{2,1, j-1 / 2}^{n^{+}}\right. \\
& \left.-\frac{D \Delta t^{n}}{\Delta x_{0} \Delta x_{1 / 2}}-\frac{D \Delta t^{n}}{\Delta y_{j} \Delta y_{j+1 / 2}}-\frac{D \Delta t^{n}}{\Delta y_{j} \Delta y_{j-1 / 2}}\right\}\left(u_{1, j}^{n}-u_{0, j}^{n}\right) \\
& +\left(\frac{\Delta t^{n}}{\Delta y_{j}} V_{2,1, j-1 / 2}^{n^{+}}+\frac{D \Delta t^{n}}{\Delta y_{j} \Delta y_{j-1 / 2}}\right)\left(u_{1, j-1}^{n}-u_{0, j-1}^{n}\right) \\
& -\left(\frac{\Delta t^{n}}{\Delta x_{1}}\left(V_{1,3 / 2, j}^{n}-V_{1,1 / 2, j}^{n}+\frac{\Delta t^{n}}{\Delta y_{j}}\left(V_{2,1, j+1 / 2}^{n}-V_{2,1, j-1 / 2}^{n}\right)\right) u_{0, j}^{n}\right. \\
+ & \left(-\frac{\Delta t^{n}}{\Delta y_{j}} V_{2,1, j+1 / 2}^{n^{-}}+\frac{D \Delta t^{n}}{\Delta y_{j} \Delta y_{j+1 / 2}}\right)\left(u_{0, j+1}^{n}-u_{0, j}^{n}\right) \\
& -\left(\frac{\Delta t^{n}}{\Delta y_{j}} V_{2,1, j-1 / 2}^{n^{+}}+\frac{D \Delta t^{n}}{\Delta y_{j} \Delta y_{j-1 / 2}}\right)\left(u_{0, j}^{n}-u_{0, j-1}^{n}\right)-K_{1, j}^{n} \Delta t^{n} u_{1, j}^{n},
\end{aligned}
$$

for $i=1, \cdots, n_{x}-1$ and $j=1, \cdots, n_{y}$,

$$
\begin{aligned}
u_{i+1, j}^{n+1}-u_{i, j}^{n+1}= & \left(-\frac{\Delta t^{n}}{\Delta x_{i+1}} V_{1, i+3 / 2, j}^{n^{-}}+\frac{D \Delta t^{n}}{\Delta x_{i+1} \Delta x_{i+3 / 2}}\right)\left(u_{i+2, j}^{n}-u_{i+1, j}^{n}\right) \\
& +\left(-\frac{\Delta t^{n}}{\Delta y_{j}} V_{2, i+1, j+1 / 2}^{n^{-}}+\frac{D \Delta t^{n}}{\Delta y_{j} \Delta y_{j+1 / 2}}\right)\left(u_{i+1, j+1}^{n}-u_{i, j+1}^{n}\right) \\
& +\left\{1-\frac{\Delta t^{n}}{\Delta x_{i+1}}\left(V_{1, i+3 / 2, j}^{n}-V_{1, i+1 / 2, j}^{n}\right)-\frac{\Delta t^{n}}{\Delta y_{j}}\left(V_{2, i+1, j+1 / 2}^{n}\right.\right.
\end{aligned}
$$




$$
\begin{aligned}
& \left.-V_{2, i+1, j-1 / 2}^{n}\right)+\frac{\Delta t^{n}}{\Delta x_{i}} V_{1, i+1 / 2, j}^{n^{-}}-\frac{\Delta t^{n}}{\Delta x_{i+1}} V_{1, i+1 / 2, j}^{n^{+}} \\
& +\frac{\Delta t^{n}}{\Delta y_{j}}\left(V_{2, i+1, j+1 / 2}^{n^{-}}-V_{2, i+1, j-1 / 2}^{n^{+}}\right)-\frac{D \Delta t^{n}}{\Delta x_{i+1 / 2}}\left(\frac{1}{\Delta x_{i}}+\frac{1}{\Delta x_{i+1}}\right) \\
& \left.+\frac{D \Delta t^{n}}{\Delta y_{j}}\left(\frac{1}{\Delta y_{j+1 / 2}}+\frac{1}{\Delta y_{j-1 / 2}}\right)-K_{i+1, j}^{n} \Delta t^{n}\right\}\left(u_{i+1, j}^{n}-u_{i, j}^{n}\right) \\
& +\left(\frac{\Delta t^{n}}{\Delta x_{i}} V_{1, i-1 / 2, j}^{n^{+}}+\frac{D \Delta t^{n}}{\Delta x_{i} \Delta x_{i-1 / 2}}\right)\left(u_{i, j}^{n}-u_{i-1, j}^{n}\right) \\
& +\left(\frac{\Delta t^{n}}{\Delta y_{j}} V_{2, i+1, j-1 / 2}^{n^{+}}+\frac{D \Delta t^{n}}{\Delta y_{j} \Delta y_{j-1 / 2}}\right)\left(u_{i+1, j-1}^{n}-u_{i, j-1}^{n}\right) \\
& -\frac{\Delta t^{n}}{\Delta y_{j}}\left(V_{2, i+1, j+1 / 2}^{n^{-}}-V_{2, i, j+1 / 2}^{n^{-}}\right)\left(u_{i, j+1}^{n}-u_{i, j}^{n}\right) \\
& +\frac{\Delta t^{n}}{\Delta y_{j}}\left(V_{2, i+1, j-1 / 2}^{n^{+}}-V_{2, i, j-1 / 2}^{n^{+}}\right)\left(u_{i, j-1}^{n}-u_{i, j}^{n}\right) \\
& -\Delta t^{n}\left(\left(\operatorname{div} V_{h}^{n}\right)_{i+1, j}-\left(\operatorname{div} V_{h}^{n}\right)_{i, j}\right) u_{i, j}^{n} \\
& +\Delta t^{n}\left(K_{i, j}^{n}-K_{i+1, j}^{n}\right) u_{i, j}^{n},
\end{aligned}
$$

and for $i=n_{x}$ and $j=1, \cdots, n_{y}$,

$$
\begin{aligned}
u_{n_{x}+1, j}^{n+1}-u_{n_{x}, j}^{n+1}= & u_{n_{x}+1, j}^{n+1}-u_{n_{x}+1, j}^{n} \\
+ & \left\{1+\frac{\Delta t^{n}}{\Delta x_{n_{x}}} V_{1, n_{x}+1 / 2, j}^{n^{-}}+\frac{\Delta t^{n}}{\Delta y_{j}} V_{2, n_{x}, j+1 / 2}^{n^{-}}-\frac{\Delta t^{n}}{\Delta y_{j}} V_{2, n_{x}, j-1 / 2}^{n^{+}}\right. \\
& -\frac{D \Delta t^{n}}{\Delta x_{n_{x}} \Delta x_{n_{x}+1 / 2}}-\frac{D \Delta t^{n}}{\Delta y_{j} \Delta y_{j+1 / 2}} \\
& \left.\quad-\frac{D \Delta t^{n}}{\Delta y_{j} \Delta y_{j-1 / 2}}\right\}\left(u_{n_{x}+1, j}^{n}-u_{n_{x}, j}^{n}\right) \\
+ & \left(\frac{\Delta t^{n}}{\Delta x_{n_{x}}} V_{1, n_{x}-1 / 2, j}^{n^{+}}+\frac{D \Delta t^{n}}{\Delta x_{n_{x}} \Delta x_{n_{x}-1 / 2}}\right)\left(u_{n_{x}, j}^{n}-u_{n_{x}-1, j}^{n}\right) \\
+ & \left(-\frac{\Delta t^{n}}{\Delta y_{j}} V_{2, n_{x}, j+1 / 2}^{n^{-}}+\frac{D \Delta t^{n}}{\Delta y_{j} \Delta y_{j+1 / 2}}\right)\left(u_{n_{x}+1, j+1}^{n}-u_{n_{x}, j+1}^{n}\right) \\
+ & \left(\frac{\Delta t^{n}}{\Delta y_{j}} V_{2, n_{x}, j-1 / 2}^{n^{+}}+\frac{D \Delta t^{n}}{\Delta y_{j} \Delta y_{j-1 / 2}}\right)\left(u_{n_{x}+1, j-1}^{n}-u_{n_{x}, j-1}^{n}\right) \\
+ & \left(\frac{\Delta t^{n}}{\Delta x_{n_{x}}}\left(V_{1, n_{x}+1 / 2, j}^{n}-V_{1, n_{x}-1 / 2, j}^{n}\right)\right. \\
& \left.+\frac{\Delta t^{n}}{\Delta y_{j}}\left(V_{1, n_{x}, j+1 / 2}^{n}-V_{1, n_{x}, j-1 / 2}^{n}\right)\right) u_{n_{x}, j}^{n} \\
+ & \left(-\frac{\Delta t^{n}}{\Delta y_{j}} V_{2, n_{x}, j+1 / 2}^{n^{-}}+\frac{D \Delta t^{n}}{\Delta y_{j} \Delta y_{j+1 / 2}}\right)\left(u_{n_{x}+1, j+1}^{n}-u_{n_{x}+1, j}^{n}\right) \\
+ & \left(\frac{\Delta t^{n}}{\Delta y_{j}} V_{2, n_{x}, j-1 / 2}^{n^{+}}+\frac{D \Delta t^{n}}{\Delta y_{j} \Delta y_{j-1 / 2}}\right)\left(u_{n_{x}+1, j}^{n}-u_{n_{x}+1, j-1}^{n}\right) \\
+ & K_{n_{x}, j}^{n} \Delta t^{n} u_{n_{x}, j}^{n} . \\
&
\end{aligned}
$$

Similar expressions hold for $u_{i, j+1}^{n+1}-u_{i, j}^{n+1}$. 
Proof. From (2.2), we see that

$$
\begin{aligned}
u_{i, j}^{n+1}= & \left(-\frac{\Delta t^{n}}{\Delta x_{i}} V_{1, i+1 / 2, j}^{n^{-}}+\frac{D \Delta t^{n}}{\Delta x_{i} \Delta x_{i+1 / 2}}\right)\left(u_{i+1, j}^{n}-u_{i, j}^{n}\right) \\
& +\left(-\frac{\Delta t^{n}}{\Delta y_{j}} V_{2, i, j+1 / 2}^{n^{-}}+\frac{D \Delta t^{n}}{\Delta y_{j} \Delta y_{j+1 / 2}}\right)\left(u_{i, j+1}^{n}-u_{i, j}^{n}\right) \\
& +\left(\frac{\Delta t^{n}}{\Delta x_{i}} V_{1, i-1 / 2, j}^{n^{+}}+\frac{D \Delta t^{n}}{\Delta x_{i} \Delta x_{i-1 / 2}}\right)\left(u_{i-1, j}^{n}-u_{i, j}^{n}\right) \\
& +\left(\frac{\Delta t^{n}}{\Delta y_{j}} V_{2, i, j-1 / 2}^{n^{+}}+\frac{D \Delta t^{n}}{\Delta y_{j} \Delta y_{j-1 / 2}}\right)\left(u_{i, j-1}^{n}-u_{i, j}^{n}\right)-K_{i, j}^{n} \Delta t^{n} u_{i, j}^{n} \\
& +\left(1-\frac{\Delta t^{n}}{\Delta x_{i}}\left(V_{1, i+1 / 2, j}^{n}-V_{1, i-1 / 2, j}^{n}\right)-\frac{\Delta t^{n}}{\Delta y_{j}}\left(V_{2, i, j+1 / 2}^{n}-V_{2, i, j-1 / 2}^{n}\right)\right) u_{i, j}^{n} .
\end{aligned}
$$

Then, the proof is completed by simple algebraic manipulations on $u_{i+1, j}^{n+1}-u_{i, j}^{n+1}$.

Lemma 5.2. Assume that

$$
0 \leq u_{i, j}^{n}, \quad i=0, \cdots, n_{x}+1, j=0, \cdots, n_{y}+1
$$

and, for $i=1, \cdots, n_{x}$ and $j=1, \cdots, n_{y}$,

$$
\Delta t^{n} \leq \frac{1}{D_{i j}^{\star}+3\left\|V_{h 1}^{n}\right\|_{L^{\infty}(\Omega)} / \Delta x_{\star}+3\left\|V_{h 2}^{n}\right\|_{L^{\infty}(\Omega)} / \Delta y_{\star}} .
$$

Then there is a constant $C_{3}=C_{3}\left(C_{0}\right)$ such that

$$
\begin{aligned}
\left\|u_{h}^{n+1}\right\|_{B V(\Omega)} \leq & \left(1+C_{3} \Delta t^{n}\left\|\nabla V^{n}\right\|_{L^{\infty}(\Omega)}\right)\left\|u_{h}^{n}\right\|_{B V(\Omega)}+\Delta t^{n}\left\|\operatorname{div} V_{h}^{n}\right\|_{B V(\Omega)} U^{n} \\
& +\Delta t^{n} C_{3}\left(\left\|\nabla V^{n}\right\|_{L^{\infty}(\Omega)}+K^{\star}\right) U^{n}+\Delta t^{n}|| K^{n} \|_{B V(\Omega)} U^{n} \\
& +2 \Delta t^{n} \sum_{j=0}^{n_{y}}\left(\left\|V_{h 2}^{n}\right\|_{L^{\infty}(\Omega)}+\frac{D}{\Delta y_{j+1 / 2}}\right)\left|u_{0, j+1}^{n}-u_{0, j}^{n}\right| \\
& +2 \Delta t^{n} \sum_{j=0}^{n_{y}}\left(\|\left. V_{h 2}^{n}\right|_{L^{\infty}(\Omega)}+\frac{D}{\Delta y_{j+1 / 2}}\right)\left|u_{n_{x}+1, j+1}^{n}-u_{n_{x}+1, j}^{n}\right| \\
& +\sum_{j=0}^{n_{y}}\left|u_{0, j}^{n+1}-u_{0, j}^{n}\right| \Delta y_{j}+\sum_{j=0}^{n_{y}}\left|u_{n_{x}+1, j}^{n+1}-u_{n_{x}+1, j}^{n}\right| \Delta y_{j} .
\end{aligned}
$$

Proof. From (5.1b) we see that the coefficients of the terms between the brackets \{\} in the expressions of Lemma 5.1 are nonnegative. Then the estimate of a typical term is given as follows:

$$
\begin{aligned}
\left|u_{i+1, j}^{n+1}-u_{i, j}^{n+1}\right| \leq & \left(-\frac{\Delta t^{n}}{\Delta x_{i+1}} V_{1, i+3 / 2, j}^{n^{-}}+\frac{D \Delta t^{n}}{\Delta x_{i+1} \Delta x_{i+3 / 2}}\right)\left|u_{i+2, j}^{n}-u_{i+1, j}^{n}\right| \\
& +\left(-\frac{\Delta t^{n}}{\Delta y_{j}} V_{2, i+1, j+1 / 2}^{n^{-}}+\frac{D \Delta t^{n}}{\Delta y_{j} \Delta y_{j+1 / 2}}\right)\left|u_{i+1, j+1}^{n}-u_{i, j+1}^{n}\right| \\
+ & \left\{1-\frac{\Delta t^{n}}{\Delta x_{i+1}}\left(V_{1, i+3 / 2, j}^{n}-V_{1, i+1 / 2, j}^{n}\right)-\frac{\Delta t^{n}}{\Delta y_{j}}\left(V_{2, i+1, j+1 / 2}^{n}\right.\right. \\
& \left.-V_{2, i+1, j-1 / 2}^{n}\right)+\frac{\Delta t^{n}}{\Delta x_{i}} V_{1, i+1 / 2, j}^{n^{-}}-\frac{\Delta t^{n}}{\Delta x_{i+1}} V_{1, i+1 / 2, j}^{n^{+}}
\end{aligned}
$$




$$
\begin{aligned}
& +\frac{\Delta t^{n}}{\Delta y_{j}}\left(V_{2, i+1, j+1 / 2}^{n^{-}}-V_{2, i+1, j-1 / 2}^{n^{+}}\right)-\frac{D \Delta t^{n}}{\Delta x_{i+1 / 2}}\left(\frac{1}{\Delta x_{i}}+\frac{1}{\Delta x_{i+1}}\right) \\
& \left.+\frac{D \Delta t^{n}}{\Delta y_{j}}\left(\frac{1}{\Delta y_{j+1 / 2}}+\frac{1}{\Delta y_{j-1 / 2}}\right)-K_{i+1, j}^{n} \Delta t^{n}\right\}\left|u_{i+1, j}^{n}-u_{i, j}^{n}\right| \\
& +\left(\frac{\Delta t^{n}}{\Delta x_{i}} V_{1, i-1 / 2, j}^{n^{+}}+\frac{D \Delta t^{n}}{\Delta x_{i} \Delta x_{i-1 / 2}}\right)\left|u_{i, j}^{n}-u_{i-1, j}^{n}\right| \\
& +\left(\frac{\Delta t^{n}}{\Delta y_{j}} V_{2, i+1, j-1 / 2}^{n^{+}}+\frac{D \Delta t^{n}}{\Delta y_{j} \Delta y_{j-1 / 2}}\right)\left|u_{i+1, j-1}^{n}-u_{i, j-1}^{n}\right| \\
& +\frac{\Delta t^{n}}{\Delta y_{j}}\left|V_{2, i+1, j+1 / 2}^{n^{-}}-V_{2, i, j+1 / 2}^{n^{-}}\right|\left|u_{i, j+1}^{n}-u_{i, j}^{n}\right| \\
& +\frac{\Delta t^{n}}{\Delta y_{j}}\left|V_{2, i+1, j-1 / 2}^{n^{+}}-V_{2, i, j-1 / 2}^{n^{+}}\right|\left|u_{i, j-1}^{n}-u_{i, j}^{n}\right| \\
& +\Delta t^{n}\left|\left(\operatorname{div} V_{h}^{n}\right)_{i+1, j}-\left(\operatorname{div} V_{h}^{n}\right)_{i, j}\right| u_{i, j}^{n} \\
& +\Delta t^{n}\left|K_{i, j}^{n}-K_{i+1, j}^{n}\right| u_{i, j}^{n},
\end{aligned}
$$

Thus simple algebraic manipulations and use of (2.3c) yield the desired result.

We are now in a position to prove Theorem 3.2.

Proof of Theorem 3.2. Note that the CFL condition (3.6) implies (5.1b) by (2.3a). Then the result (3.7) follows by iterating on $n$ the inequality in Lemma 5.2 and using Theorem 3.1 and $(2.3 \mathrm{~b})$.

6. Proof of continuity with respect to data. In this section we prove Theorem 3.3 and a result on equicontinuity in time of the approximate solution, Proposition 6.4 below. We recall that $v_{h}$ stands for the solution of (2.2) with the data $v_{D}$ and $v_{\text {init }}$.

LEMMA 6.1. For $n=0, \cdots, n_{T}, i=1, \cdots, n_{x}$, and $j=1, \cdots, n_{y}$, we have

$$
\begin{aligned}
u_{i, j}^{n+1}-v_{i, j}^{n+1}= & A_{i+1, j}^{n}\left(u_{i+1, j}^{n}-v_{i+1, j}^{n}\right)+A_{i, j+1}^{n}\left(u_{i, j+1}^{n}-v_{i, j+1}^{n}\right) \\
& +B_{i, j}^{n}\left(u_{i, j}^{n}-v_{i, j}^{n}\right)+E_{i-1, j}^{n}\left(u_{i-1, j}^{n}-v_{i-1, j}^{n}\right) \\
& +E_{i, j-1}^{n}\left(u_{i, j-1}^{n}-v_{i, j-1}^{n}\right)
\end{aligned}
$$

where $A_{i+1, j}^{n}, A_{i, j+1}^{n}, B_{i, j}^{n}, E_{i-1, j}^{n}$, and $E_{i, j-1}^{n}$ are defined as in the proof of Lemma 4.1 .

The result easily follows from (2.2).

Lemma 6.2. Supposed that (4.1) is satisfied. Then

$$
\begin{aligned}
\left\|u_{h}^{n+1}-v_{h}^{n+1}\right\|_{L^{1}(\Omega)} \leq \Delta t^{n} & \left(\left\|V_{h 1}^{n}\right\|_{L^{\infty}(\Omega)}+\left\|V_{h 2}^{n}\right\|_{L^{\infty}(\Omega)}+D\left(\frac{1}{\Delta x_{\star}}+\frac{1}{\Delta y_{\star}}\right)\right) \\
& \times\left\|u_{D}-v_{D}\right\|_{L^{1}\left(\partial \Omega_{2}\right)}+\left\|u_{h}^{n}-v_{h}^{n}\right\|_{L^{1}(\Omega)} .
\end{aligned}
$$

Proof. Since, by (4.1), the coefficients in the equality of Lemma 6.1 are nonnegative, we see that

$$
\begin{aligned}
\left|u_{i, j}^{n+1}-v_{i, j}^{n+1}\right| \leq & A_{i+1, j}^{n}\left|u_{i+1, j}^{n}-v_{i+1, j}^{n}\right|+A_{i, j+1}^{n}\left|u_{i, j+1}^{n}-v_{i, j+1}^{n}\right| \\
& +B_{i, j}^{n}\left|u_{i, j}^{n}-v_{i, j}^{n}\right|+E_{i-1, j}^{n}\left|u_{i-1, j}^{n}-v_{i-1, j}^{n}\right| \\
& +E_{i, j-1}^{n}\left|u_{i, j-1}^{n}-v_{i, j-1}^{n}\right| .
\end{aligned}
$$

Then multiplying by $\Delta x_{i} \Delta y_{j}$, adding over $i, j$, and rearranging terms imply the desired result. 
Now Theorem 3.3 can be easily seen from Lemma 6.2 .

Lemma 6.3. Assume that the CFL condition (4.1) is satisfied. Then

$$
\begin{aligned}
\left\|u_{h}^{1}-u_{h}^{0}\right\|_{L^{1}(\Omega)} \leq & 2 \Delta t^{0}\left(\left\|V_{h 1}^{0}\right\|_{L^{\infty}(\Omega)}+\left\|V_{h 2}^{0}\right\|_{L^{\infty}(\Omega)}\right. \\
& \left.+D\left(\frac{1}{\Delta x_{\star}}+\frac{1}{\Delta y_{\star}}\right)\right)\left\|u_{\text {init }}\right\|_{B V(\Omega)} \\
& +\Delta t^{0}\left(\left\|V_{h 1}^{0}\right\|_{B V(\Omega)}+\left\|V_{h 2}^{0}\right\|_{B V(\Omega)}+K^{\star}\right) u^{\star} .
\end{aligned}
$$

Proof. By (2.2), we observe that

$$
\begin{aligned}
\left|u_{i, j}^{1}-u_{i, j}^{0}\right| \leq & \left(-\frac{\Delta t^{0}}{\Delta x_{i}} V_{1, i+1 / 2, j}^{0^{-}}+\frac{D \Delta t^{0}}{\Delta x_{i} \Delta x_{i+1 / 2}}\right)\left|u_{i+1, j}^{0}-u_{i, j}^{0}\right| \\
& +\left(-\frac{\Delta t^{0}}{\Delta y_{j}} V_{2, i, j+1 / 2}^{0^{-}}+\frac{D \Delta t^{0}}{\Delta y_{j} \Delta y_{j+1 / 2}}\right)\left|u_{i, j+1}^{0}-u_{i, j}^{0}\right| \\
& +\left(\frac{\Delta t^{0}}{\Delta x_{i}} V_{1, i-1 / 2, j}^{0^{+}}+\frac{D \Delta t^{0}}{\Delta x_{i} \Delta x_{i-1 / 2}}\right)\left|u_{i-1, j}^{0}-u_{i, j}^{0}\right| \\
& +\left(\frac{\Delta t^{0}}{\Delta y_{j}} V_{2, i, j-1 / 2}^{0^{+}}+\frac{D \Delta t^{0}}{\Delta y_{j} \Delta y_{j-1 / 2}}\right)\left|u_{i, j-1}^{0}-u_{i, j}^{0}\right|+\Delta t^{0} K_{i, j}^{0} u_{i, j}^{0} \\
& +\Delta t^{0}\left|\frac{1}{\Delta x_{i}}\left(V_{1, i+1 / 2, j}^{0}-V_{1, i-1 / 2, j}^{0}\right)+\frac{1}{\Delta y_{j}}\left(V_{2, i, j+1 / 2}^{0}-V_{2, i, j-1 / 2}^{0}\right)\right| u_{i, j}^{0} .
\end{aligned}
$$

Then the lemma follows by multiplying this inequality by $\Delta x_{i} \Delta y_{j}$ and adding the resulting one over $i, j$.

Proposition 6.4 (EquiContinUity in TIME). Under the assumptions of Theorem 3.1, there is a constant $C_{4}$ depending only on the data and $T$ such that for $n=0, \cdots, n_{T}$

$$
\begin{aligned}
\left\|u_{h}^{n+1}-u_{h}^{n}\right\|_{L^{1}(\Omega)} \leq C_{4} \Delta t & \left(1+D\left(\frac{1}{\Delta x_{\star}}+\frac{1}{\Delta y_{\star}}\right)\right) \\
& \times\left(\left\|u_{\text {init }}\right\|_{B V(\Omega)}+\left\|u_{D}\right\|_{L^{1}\left(\partial \Omega_{2} ; B V(J)\right)}\right) .
\end{aligned}
$$
sult.

Proof. Take $v_{h}^{n+1}=u_{h}^{n}$ in Lemma 6.2 and use Lemma 6.3 to obtain the re-

7. A convergence analysis. In this section we prove Theorem 3.4 by applying the ideas used in [10] for analyzing the one-dimensional drift-diffusion semiconductor device equations. We point out that the analysis here is much simpler than that given in [10]. The reason is that we are here using the standard entropy $|\cdot|$, while a smoother entropy has been used there, which requires muck work to estimate the distance between the smooth entropy and the standard one. We also emphasize the difference between the present analysis and that used in classical conservation laws; in the present case the delicate part is how to handle the boundary terms in the 'entropy form' $\Theta$ (see (7.3) below), while an unbounded domain is treated in the classical conservation laws.

It should be emphasized that this whole section concerns the case of $D=0$, and that, although the differential equation (1.1) is linear, techniques which have been originally developed for nonlinear hyperbolic conservation laws will be used.

The proof of Theorem 3.4 proceeds as follows. First, we prove that there is a 
subsequence $\left\{u_{h^{\prime}}\right\}_{h^{\prime}>0}$ converging to a limit $u$. Then, we show that

$$
\begin{aligned}
& \lim _{h^{\prime} \rightarrow 0} \mathcal{R}\left(u_{h^{\prime}}, \varphi\right)=\mathcal{R}(u, \varphi), \\
& \mathcal{R}(u, \varphi)=0,
\end{aligned}
$$

for $\varphi \in \mathcal{C}_{0}^{1}([0, T) \times \bar{\Omega})$, where $\mathcal{R}(\cdot, \cdot)$ defines the left-hand side of (3.10). Since the weak solution of (3.10) is assumed unique, this completes the proof of Theorem 3.4.

As in classical conservation laws, (7.1) follows from the following result [13]:

$$
\begin{aligned}
& \lim _{h^{\prime} \rightarrow 0} \Theta\left(u_{h^{\prime}}, c ; V_{h^{\prime}} ; \varphi\right)=\Theta(u, c ; V ; \varphi), \quad \forall c \in \mathbb{R}, \varphi \in \mathcal{C}^{1}\left(\bar{Q}_{T}\right), \\
& \Theta(u, c ; V ; \varphi) \leq 0, \quad \forall c \in \mathbb{R}, 0 \leq \varphi \in \mathcal{C}^{1}\left(\bar{Q}_{T}\right),
\end{aligned}
$$

where $\Theta$ is defined in (7.3) below. Most part of this section is devoted to proving this result.

7.1. The entropy form. The entropy form $\Theta(u, c ; V ; \varphi)$ with boundary terms included is defined as follows:

$$
\begin{aligned}
\Theta(u, c ; V ; \varphi)= & -\left(|u-c|, \varphi_{t}\right)_{Q_{T}}-(|u-c| V, \nabla \varphi)_{Q_{T}} \\
& +\left.(|u-c|, \varphi)\right|_{\{t=T\} \times \Omega}-\left.\left(\left|u_{\text {init }}-c\right|, \varphi\right)\right|_{\{t=0\} \times \Omega} \\
& +\left(G\left(u-c, u_{D}-c ; V \cdot \nu\right), \varphi\right)_{J \times(\partial \Omega)} \\
& -(H(u, c)(\operatorname{div} V+K), \varphi)_{Q_{T}}+(K|u-c|, \varphi)_{Q_{T}},
\end{aligned}
$$

where $c \in \mathbb{R}, \varphi \in \mathcal{C}^{1}\left(\bar{Q}_{T}\right)$, and the 'entropy flux' $G$ and the function $H$ are defined by

$$
\begin{aligned}
& G\left(u_{\text {left }}, u_{\text {right }} ; V \cdot \nu\right)=\left|u_{\text {left }}\right|(V \cdot \nu)^{+}+\left|u_{\text {right }}\right|(V \cdot \nu)^{-}, \\
& H(u, c)=|u-c|-u \operatorname{sign}(u-c) .
\end{aligned}
$$

The motivation of the form $\Theta$ can be given as in the one-dimensional case [10].

7.2. A convergent subsequence. In this subsection we prove the existence of a convergent subsequence $\left\{u_{h^{\prime}}\right\}_{h^{\prime}>0}$.

Lemma 7.1. Assume that the hypotheses of Theorem 3.2 are satisfied. Then there exists a subsequence $\left\{u_{h^{\prime}}\right\}_{h^{\prime}>0}$ converging in $L^{\infty}\left(J ; L^{1}(\Omega)\right)$ to a limit $u$ in $L^{\infty}(J ; B V(\Omega)) \cap \mathcal{C}^{0}\left(J ; L^{1}(\Omega)\right)$.

Proof. We note that the ideas in [13] can be used to prove the lemma. In [13], a discrete version of Azcoli-Arzelá Theorem was used. In the present case with $D=0$, the equicontinuity in time is provided by Proposition 6.4, and the compactness of the range is given by Theorem 3.2. Also, the regularity result on $u$ follows from the convergence and Theorem 3.2.

7.3. Proof of (7.2a). Here we prove (7.2a) under a condition.

Lemma 7.2. Suppose that for $c \in \mathbb{R}$ and nonnegative $\varphi \in \mathcal{C}^{1}\left(\bar{Q}_{T}\right)$,

$$
\lim _{h^{\prime} \rightarrow 0} \Theta\left(u_{h^{\prime}}, c ; V_{h^{\prime}} ; \varphi\right) \leq 0 .
$$

Then

$$
\lim _{h^{\prime} \rightarrow 0} \Theta\left(u_{h^{\prime}}, c ; V_{h^{\prime}} ; \varphi\right)=\Theta(u, c ; V ; \varphi) .
$$


Proof. First, for every nonnegative $\varphi \in \mathcal{C}_{0}^{1}([0, T) \times \Omega),(7.5)$ follows from Lemma 7.1 and the standard argument in the classical conservation laws [13]. Also, since $u \in \mathcal{C}^{0}\left(J ; L^{1}(\Omega)\right)$ by Lemma 7.1 , the same result holds for $\varphi \in \mathcal{C}_{0}^{1}([0, T] \times \Omega)$.

We now consider the case where $\varphi \in \mathcal{C}_{0}^{1}(J \times[0,1) \times(0,1))$. Since we are mainly concerned with the boundary term associated with the edge $\{x=0,0<y<1\}$, it suffices to consider $\varphi(t, x, y)$ of this form $\omega(t, y) \xi(x)$. Then, set

$$
\begin{aligned}
g_{h^{\prime}}(x) & =\int_{0}^{T} \int_{0}^{1}\left|u_{h^{\prime}}-c\right| V_{h^{\prime} 1} \omega(t, y) d y d t, \\
g_{h^{\prime}}(0-) & =\int_{0}^{T} \int_{0}^{1}\left\{\left|u_{D, \Delta t}-c\right| V_{h^{\prime} 1}^{+}(t, 0, y)+\left|u_{h^{\prime}}(t, 0+, y)-c\right| V_{h^{\prime} 1}^{-}(t, 0, y)\right\} \omega d t d y,
\end{aligned}
$$

and rewrite $\Theta\left(u_{h^{\prime}}, c ; V_{h^{\prime}} ; \varphi\right)$ as follows:

$$
\begin{aligned}
\Theta\left(u_{h^{\prime}}, c ; \varphi\right)= & -\left(\left|u_{h^{\prime}}-c\right|, \xi \frac{\partial \omega}{\partial t}\right)_{Q_{T}}-\left(\left|u_{h^{\prime}}-c\right| V_{h^{\prime}}, \xi \frac{\partial \omega}{\partial y}\right)_{Q_{T}} \\
& -\int_{0}^{1} g_{h^{\prime}}(x) \xi^{\prime}(x) d x-g_{h^{\prime}}(0-) \xi(0) \\
& -\left(H\left(u_{h^{\prime}}, c\right)\left(\operatorname{div} V_{h^{\prime}}+K\right), \xi \omega\right)_{Q_{T}}+\left(K\left|u_{h^{\prime}}-c\right|, \xi \omega\right)_{Q_{T}} .
\end{aligned}
$$

Since the sequence $\left\{u_{h^{\prime}}(\cdot, 0+, \cdot)\right\}_{h^{\prime}>0}$ is bounded in $L^{\infty}(J \times(0,1))$ by Theorem 3.1, there is a subsequence $\left\{u_{h^{\prime \prime}}(\cdot, 0+, \cdot)\right\}_{h^{\prime \prime}>0}$ converging in $L^{\infty}(J \times(0,1))$-weak ${ }^{\star}$ to a limit $\widetilde{u}$. Let $\gamma_{t, y}$ be the Young measure corresponding to $\widetilde{u}$. Then, by Lemma 7.1 and (2.3a), we see that

$$
\begin{aligned}
\lim _{h^{\prime \prime} \rightarrow 0} \Theta\left(u_{h^{\prime \prime}}, c ; V_{h^{\prime}} ; \varphi\right)= & -\left(|u-c|, \xi \frac{\partial \omega}{\partial t}\right)_{Q_{T}}-\left(|u-c| V_{2}, \xi \frac{\partial \omega}{\partial y}\right)_{Q_{T}} \\
& -\int_{0}^{1} g(x) \xi^{\prime}(x) d x-g_{0} \xi(0) \\
& -(H(u, c)(\operatorname{div} V+K), \xi \omega)_{Q_{T}}+(K|u-c|, \xi \omega)_{Q_{T}},
\end{aligned}
$$

where

$$
\begin{aligned}
& g(x)=\int_{0}^{T} \int_{0}^{1}|u-c| V_{1} \omega(t, y) d y d t \\
& g_{0}=\int_{0}^{T} \int_{0}^{1}\left\{\left|u_{D}-c\right| V_{1}^{+}(t, 0, y)+w(t, y) V_{1}^{-}(t, 0, y)\right\} \omega d t d y
\end{aligned}
$$

where

$$
\begin{aligned}
w(t, y) & =\int_{0}^{u^{\star \star}}|\lambda-c| d \gamma_{t, y}(\lambda), \\
u^{\star \star} & =\mathrm{e}^{T V_{D}^{\star}} u^{\star} .
\end{aligned}
$$

Thus, to prove (7.5), it suffices to prove that $g_{0}=g^{\star}$ where

$$
g^{\star}=\int_{0}^{T} \int_{0}^{1}\left\{\left|u_{D}-c\right| V_{1}^{+}(t, 0, y)+|u(t, 0+, y)-c| V_{1}^{-}(t, 0, y)\right\} \omega d t d y .
$$

Take $\xi$ such that its support is contained in $[0, \epsilon]$. Then, by (7.4) and Theorem 3.1, it 
follows from (7.6) and (7.7) that

$$
-\int_{0}^{1} g(x) \xi^{\prime}(x) d x-g_{0} \xi(0) \leq C \epsilon\|\xi\|_{L^{1}(0,1)} .
$$

Since $\epsilon$ is arbitrary, this inequality yields that

$$
g(0+)-g_{0} \leq 0 .
$$

Choose $c \in \mathbb{R}$ such that $|u-c|=\alpha(u-c)$ for some $\alpha \in \mathbb{R}$. Then, by (7.8) and the definition of $\gamma_{t, y}$,

$$
w(t, y)=\alpha(\widetilde{u}-c)
$$

so that, by (7.7), (7.10) becomes

$$
\begin{aligned}
\alpha\left\{\int_{0}^{T} \int_{0}^{1}\left(u(t, 0+, y)-u_{D}\right) V_{1}^{+}(t, 0, y) \omega d y d t\right. & \\
& \left.+(u(t, 0+, y)-\widetilde{u}) V_{1}^{-}(t, 0, y) \omega d y d t\right\} \leq 0 .
\end{aligned}
$$

Since the sign of $\alpha$ is arbitrary and this inequality is true for any nonnegative $\omega \in$ $\mathcal{C}_{0}^{1}(T \times(0,1))$, we have

$$
\begin{aligned}
& V_{1}^{+}(t, 0, y)\left(u(t, 0+, y)-u_{D}\right)=0, \quad \text { a.e. in } J \times(0,1), \\
& V_{1}^{-}(t, 0, y)(u(t, 0+, y)-\widetilde{u})=0, \quad \text { a.e. in } J \times(0,1) .
\end{aligned}
$$

Finally, by (7.7)-(7.9) and (7.11), we see that

$$
\begin{aligned}
g(0+)-g_{0} & =g^{\star}-g_{0} \\
& =\int_{0}^{T} \int_{0}^{1}(|u(t, 0+, y)-c|-w(t, y)) V_{1}^{-}(t, 0, y) \omega d y d t \\
& =\int_{0}^{T} \int_{0}^{1}(|\widetilde{u}(t, y)-c|-w(t, y)) V_{1}^{-}(t, 0, y) \omega d y d t \\
& =\int_{0}^{T} \int_{0}^{1}\left(\left|\int_{0}^{u^{\star \star}}(\lambda-c) d \gamma_{t, y}(\lambda)\right|-w(t, y)\right) V_{1}^{-}(t, 0, y) \omega d y d t \\
& \geq \int_{0}^{T} \int_{0}^{1}\left(\int_{0}^{u^{\star \star}}|\lambda-c| d \gamma_{t, y}(\lambda)-w(t, y)\right) V_{1}^{-}(t, 0, y) \omega d y d t \\
& =0
\end{aligned}
$$

which together with (7.10) implies that $g_{0}=g^{\star}$. This completes the proof of the case where $\varphi \in \mathcal{C}_{0}^{1}(J \times[0,1) \times(0,1))$. The same argument applies to the remaining three cases.

It is now clear that it suffices to prove (7.4). This is done in the next two subsections.

7.4. A discrete entropy inequality. The following discrete entropy inequality will be needed for obtaining an upper bound for $\Theta\left(u_{h^{\prime}}, c ; V_{h^{\prime}} ; \varphi\right)$. 
Lemma 7.3. Under the CFL condition (3.2), we have, for $c \in \mathbb{R}$,

$$
\begin{aligned}
& \left|u_{i, j}^{n+1}-c\right|-\left(1-K_{i, j}^{n} \Delta t^{n}\right)\left|u_{i, j}^{n}-c\right|+\frac{\Delta t^{n}}{\Delta x_{i}}\left(G_{i+1 / 2, j}^{n}-G_{i-1 / 2, j}^{n}\right) \\
& \quad+\frac{\Delta t^{n}}{\Delta y_{j}}\left(G_{i, j+1 / 2}^{n}-G_{i, j-1 / 2}^{n}\right)-H\left(u_{i, j}^{n+1}, c\right)\left\{\left(\operatorname{div} V_{h}^{n}\right)_{i, j}+K_{i, j}^{n}\right\} \Delta t^{n} \leq 0,
\end{aligned}
$$

where

$$
\begin{aligned}
& G_{i+1 / 2, j}^{n}=V_{1, i+1 / 2, j}^{n^{+}}\left|u_{i, j}^{n}-c\right|+V_{1, i+1 / 2, j}^{n^{-}}\left|u_{i+1, j}^{n}-c\right|, \\
& G_{i, j+1 / 2}^{n}=V_{2, i, j+1 / 2}^{n^{+}}\left|u_{i, j}^{n}-c\right|+V_{2, i, j+1 / 2}^{n^{-}}\left|u_{i, j+1}^{n}-c\right| .
\end{aligned}
$$

Proof. From (2.2) and the definition of the mixed finite element space $Q_{h}$, we have, for $c \in \mathbb{R}$,

$$
\begin{aligned}
u_{i, j}^{n+1}-c= & \left(-\frac{\Delta t^{n}}{\Delta x_{i}} V_{1, i+1 / 2, j}^{n^{-}}\right)\left(u_{i+1, j}^{n}-c\right)+\left(-\frac{\Delta t^{n}}{\Delta y_{j}} V_{2, i, j+1 / 2}^{n^{-}}\right)\left(u_{i, j+1}^{n}-c\right) \\
& +\left\{1-\frac{\Delta t^{n}}{\Delta x_{i}}\left(V_{1, i+1 / 2, j}^{n^{+}}-V_{1, i-1 / 2, j}^{n^{-}}\right)-\frac{\Delta t^{n}}{\Delta y_{j}}\left(V_{2, i, j+1 / 2}^{n^{+}}-V_{2, i, j-1 / 2}^{n^{-}}\right)\right. \\
& +\left(\frac{\Delta t^{n}}{\Delta x_{i}} V_{1, i-1 / 2, j}^{n^{+}}\right)\left(K_{i, j}^{n} \Delta t^{n}\right\}\left(u_{i, j}^{n}-c\right) \\
& -\Delta t^{n}\left[\left(\operatorname{div} V_{h}^{n}\right)_{i, j}+K_{i, j}^{n}\right] c .
\end{aligned}
$$

Note that the term between the brackets is nonnegative by (3.2). Thus the lemma follows by multiplying this expression by $\operatorname{sign}\left(u_{i, j}^{n+1}-c\right)$.

7.5. An upper bound of entropy form. In this section we obtain an upper bound for $\Theta\left(u_{h^{\prime}}, c ; V_{h^{\prime}} ; \varphi\right)$, which implies the inequality (7.4). We first have the following decomposition of $\Theta\left(u_{h^{\prime}}, c ; V_{h^{\prime}} ; \varphi\right)$.

Let, for $\varphi \in C^{1}\left(\bar{Q}_{T}\right)$,

$$
\begin{aligned}
& \varphi_{i, j}^{n}=\frac{1}{\Delta x_{i}} \frac{1}{\Delta y_{j}} \int_{x_{i-1 / 2}}^{x_{i+1 / 2}} \int_{y_{j-1 / 2}}^{y_{j+1 / 2}} \varphi\left(t^{n}, x, y\right) d x d y \\
& \varphi_{i, j}^{n+1 / 2}=\frac{1}{\Delta t^{n}} \frac{1}{\Delta x_{i}} \frac{1}{\Delta y_{j}} \int_{t^{n}}^{t^{n+1}} \int_{x_{i-1 / 2}}^{x_{i+1 / 2}} \int_{y_{j-1 / 2}}^{y_{j+1 / 2}} \varphi(t, x, y) d t d x d y, \\
& \varphi_{i+1 / 2, j}^{n+1 / 2}=\frac{1}{\Delta t^{n}} \frac{1}{\Delta y_{j}} \int_{t^{n}}^{t^{n+1}} \int_{y_{j-1 / 2}}^{y_{j+1 / 2}} \varphi\left(t, x_{i+1 / 2}, y\right) d t d y, \\
& \varphi_{i, j+1 / 2}^{n+1 / 2}=\frac{1}{\Delta t^{n}} \frac{1}{\Delta x_{i}} \int_{t^{n}}^{t^{n+1}} \int_{y_{j-1 / 2}}^{y_{j+1 / 2}} \varphi\left(t, x, y_{j+1 / 2}\right) d t d x .
\end{aligned}
$$

Lemma 7.4. (DeComposition of $\Theta$ ). We have

$$
\Theta\left(u_{h}, c ; V_{h^{\prime}} ; \varphi\right)=\Theta_{e n t}\left(u_{h}, c ; V_{h^{\prime}} ; \varphi\right)+\Theta_{c o m}\left(u_{h}, c ; V_{h^{\prime}} ; \varphi\right),
$$


where (with arguments omitted)

$$
\begin{aligned}
\Theta_{e n t}= & \sum_{n=0}^{n_{T}-1} \sum_{i=1}^{n_{x}} \sum_{j=1}^{n_{y}}\left\{\left|u_{i, j}^{n+1}-c\right|-\left(1-K_{i, j}^{n} \Delta t^{n}\right)\left|u_{i, j}^{n}-c\right|\right. \\
& +\frac{\Delta t^{n}}{\Delta x_{i}}\left(G_{i+1 / 2, j}^{n}-G_{i-1 / 2, j}^{n}\right)+\frac{\Delta t^{n}}{\Delta y_{j}}\left(G_{i, j+1 / 2}^{n}-G_{i, j-1 / 2}^{n}\right) \\
& \left.-H\left(u_{i, j}^{n+1}, c\right)\left[\left(\operatorname{div} V_{h}^{n}\right)_{i, j}+K_{i, j}^{n}\right] \Delta t^{n}\right\} \varphi_{i, j}^{n+1} \Delta x_{i} \Delta y_{j},
\end{aligned}
$$

and

$$
\begin{aligned}
\Theta_{c o m}= & \sum_{n=0}^{n_{T}-1} \sum_{i=1}^{n_{x}} \sum_{j=1}^{n_{y}}\left\{\left(\left|u_{i+1, j}^{n}-c\right|-\left|u_{i, j}^{n}-c\right|\right)\left(-V_{1, i+1 / 2, j}^{n^{-}}\right)\left(\varphi_{i, j}^{n+1}-\varphi_{i+1 / 2, j}^{n+1 / 2}\right)\right. \\
& \left.+\left(\left|u_{i-1, j}^{n}-c\right|-\left|u_{i, j}^{n}-c\right|\right)\left(V_{1, i-1 / 2, j}^{n^{+}}\right)\left(\varphi_{i, j}^{n+1}-\varphi_{i-1 / 2, j}^{n+1 / 2}\right)\right\} \Delta t^{n} \Delta y_{j} \\
& +\sum_{n=0}^{n_{T}-1} \sum_{i=1}^{n_{x}} \sum_{j=1}^{n_{y}}\left\{\left(\left|u_{i, j+1}^{n}-c\right|-\left|u_{i, j}^{n}-c\right|\right)\left(-V_{2, i, j+1 / 2}^{n^{-}}\right)\left(\varphi_{i, j}^{n+1}-\varphi_{i, j+1 / 2}^{n+1 / 2}\right)\right. \\
& +\sum_{n=0}^{n_{T}-1} \sum_{i=1}^{n_{x}} \sum_{j=1}^{n_{y}}\left(\operatorname{div} V_{h}^{n}\right)_{i, j} u_{i, j}^{n} \operatorname{sign}\left(u_{i, j}^{n}-c\right)\left(\varphi_{i, j}^{n+1}-\varphi_{i, j}^{n+1 / 2}\right) \Delta t^{n} \Delta x_{i} \Delta y_{j} \\
& +\sum_{n=0}^{n_{T}-1} \sum_{i=1}^{n_{x}} \sum_{j=1}^{n_{y}} K_{i, j}^{n} H\left(u_{i, j}^{n+1}, c\right)\left(\varphi_{i, j}^{n+1}-\varphi_{i, j}^{n+1 / 2}\right) \Delta t^{n} \Delta x_{i} \Delta y_{j} \\
& +\sum_{n=0}^{n_{T}-1} \sum_{i=1}^{n_{x}} \sum_{j=1}^{n_{y}} K_{i, j}^{n}\left|u_{i, j}^{n}-c\right|\left(\varphi_{i, j}^{n}-\varphi_{i, j}^{n+1}\right) \Delta t^{n} \Delta x_{i} \Delta y_{j} \\
& +\sum_{n=0}^{n_{T}-1} \sum_{i=1}^{n_{x}} \sum_{j=1}^{n_{y}}\left(\operatorname{div} V_{h}^{n}\right)_{i, j}\left(H\left(u_{i, j}^{n+1}, c\right)-H\left(u_{i, j}^{n}, c\right)\right) \varphi_{i, j}^{n+1} \Delta t^{n} \Delta x_{i} \Delta y_{j} \\
& +\sum_{n=0}^{n_{T}-1} \sum_{i=1}^{n_{x}} \sum_{j=1}^{n_{y}} K_{i, j}^{n}\left(H\left(u_{i, j}^{n+1}, c\right)-H\left(u_{i, j}^{n}, c\right)\right) \varphi_{i, j}^{n+1 / 2} \Delta t^{n} \Delta x_{i} \Delta y_{j} .
\end{aligned}
$$

Proof. From the definition of $\Theta$ and the fact that $\operatorname{div} V_{h}$ is piecewise constant, we have

$$
\Theta=\Psi_{t}+\Psi_{x}+\Psi
$$

where

$$
\begin{aligned}
\Psi_{t}= & -\sum_{n=0}^{n_{T}-1} \sum_{i=1}^{n_{x}} \sum_{j=1}^{n_{y}}\left|u_{i, j}^{n}-c\right|\left(\varphi_{i, j}^{n+1}-\varphi_{i, j}^{n}\right) \Delta x_{i} \Delta y_{j}+\sum_{i=1}^{n_{x}} \sum_{j=1}^{n_{y}}\left|u_{i, j}^{n_{T}}-c\right| \varphi_{i, j}^{n_{T}} \Delta x_{i} \Delta y_{j} \\
& -\sum_{i=1}^{n_{x}} \sum_{j=1}^{n_{y}}\left|u_{i, j}^{0}-c\right| \varphi_{i, j}^{0} \Delta x_{i} \Delta y_{j}
\end{aligned}
$$


18

$$
\begin{aligned}
\Psi_{x}= & -\sum_{n=0}^{n_{T}-1} \sum_{i=1}^{n_{x}} \sum_{j=1}^{n_{y}}\left|u_{i, j}^{n}-c\right|\left(V_{1, i+1 / 2, j}^{n} \varphi_{i+1 / 2, j}^{n+1 / 2}-V_{1, i-1 / 2, j}^{n} \varphi_{i-1 / 2, j}^{n+1 / 2}\right) \Delta t^{n} \Delta y_{j} \\
& -\sum_{n=0}^{n_{T}-1} \sum_{i=1}^{n_{x}} \sum_{j=1}^{n_{y}}\left|u_{i, j}^{n}-c\right|\left(V_{2, i, j+1 / 2}^{n} \varphi_{i, j+1 / 2}^{n+1 / 2}-V_{2, i, j-1 / 2}^{n} \varphi_{i, j-1 / 2}^{n+1 / 2}\right) \Delta t^{n} \Delta x_{i} \\
& +\sum_{n=0}^{n_{T}-1} \sum_{j=1}^{n_{y}} G_{n_{x}+1 / 2, j}^{n} \varphi_{n_{x}+1 / 2, j}^{n+1 / 2} \Delta t^{n} \Delta y_{j}-\sum_{n=0}^{n_{T}-1} \sum_{j=1}^{n_{y}} G_{1 / 2, j}^{n} \varphi_{1 / 2, j}^{n+1 / 2} \Delta t^{n} \Delta y_{j} \\
& +\sum_{n=0}^{n_{T}-1} \sum_{i=1}^{n_{x}} G_{i, n_{y}+1 / 2}^{n_{2}} \varphi_{i, n_{y}+1 / 2}^{n+1 / 2} \Delta t^{n} \Delta x_{i}-\sum_{n=0}^{n_{T}-1} \sum_{i=1}^{n_{x}} G_{i, 1 / 2}^{n} \varphi_{i, 1 / 2}^{n+1 / 2} \Delta t^{n} \Delta x_{i} \\
\Psi= & -\sum_{n=0}^{n_{T}-1} \sum_{i=1}^{n_{x}} \sum_{j=1}^{n_{y}}\left(\operatorname{div} V_{h}^{n}\right)_{i, j} u_{i, j}^{n} \operatorname{sign}\left(u_{i, j}^{n}-c\right) \varphi_{i, j}^{n+1 / 2} \Delta t^{n} \Delta x_{i} \Delta y_{j} \\
& -\sum_{n=0}^{n_{T}-1} \sum_{i=1}^{n_{x}} \sum_{j=1}^{n_{y}} K_{i, j}^{n} H\left(u_{i, j}^{n}, c\right) \varphi_{i, j}^{n} \Delta t^{n} \Delta x_{i} \Delta y_{j} \\
& +\sum_{n=0}^{n_{T}-1} \sum_{i=1}^{n_{x}} \sum_{j=1}^{n_{y}} K_{i, j}^{n}\left|u_{i, j}^{n}-c\right| \varphi_{i, j}^{n} \Delta t^{n} \Delta x_{i} \Delta y_{j} .
\end{aligned}
$$

Then simple algebraic manipulations yield the desired result.

Lemma 7.5. (UPPER BOUnd of $\Theta$ ). Suppose that the conditions of Theorem 3.2 are satisfied. Then there is a constant $C_{5}$ depending solely on the data and $T$ such that for any $\varphi \in \mathcal{C}^{1}\left(\bar{Q}_{T}\right), \varphi \geq 0$ :

$$
\begin{aligned}
& \Theta_{\text {ent }} \leq 0 \\
& \Theta_{\text {com }} \leq C_{5}(1+|c|)\left\{\begin{aligned}
\Delta & x\left\|\varphi_{x}\right\|_{L^{\infty}\left(Q_{T}\right)}+\Delta y\left\|\varphi_{y}\right\|_{L^{\infty}\left(Q_{T}\right)} \\
& \left.+\Delta t\left(\left\|\varphi_{t}\right\|_{L^{\infty}\left(Q_{T}\right)}+\|\varphi\|_{L^{\infty}\left(Q_{T}\right)}\right)\right\} .
\end{aligned}\right.
\end{aligned}
$$

Proof. The first inequality follows immediately from Lemmas 7.3 and 7.4. Also, observe that

$$
\begin{aligned}
& \left|\varphi_{i, j}^{n+1}-\varphi_{i+1 / 2, j}^{n+1 / 2}\right| \leq \frac{1}{2} \Delta x_{i}|| \varphi_{x}\left\|_{L^{\infty}(J \times \Omega)}+\frac{1}{2} \Delta t^{n}|| \varphi_{t}\right\|_{L^{\infty}(J \times \Omega)}, \\
& \left|\varphi_{i, j}^{n+1}-\varphi_{i, j+1 / 2}^{n+1 / 2}\right| \leq \frac{1}{2} \Delta y_{j}|| \varphi_{y}\left\|_{L^{\infty}(J \times \Omega)}+\frac{1}{2} \Delta t^{n}\right\| \varphi_{t} \|_{L^{\infty}(J \times \Omega)}, \\
& \left|\varphi_{i, j}^{n+1}-\varphi_{i, j}^{n+1 / 2}\right| \leq \frac{1}{2} \Delta t^{n}|| \varphi_{t} \|_{L^{\infty}(J \times \Omega)}, \\
& \left|\varphi_{i, j}^{n+1}-\varphi_{i, j}^{n}\right| \leq \Delta t^{n}|| \varphi_{t} \|_{L^{\infty}(J \times \Omega)} .
\end{aligned}
$$

Then, if an integration by parts on $n$ is applied to the last two terms in the expression of $\Theta_{\text {com }}$, the second inequality follows from Theorems 3.1 and 3.2, Proposition 6.4, Lemma 7.4 , and (2.3a).

We are now in a position to prove Theorem 3.4.

Proof of Theorem 3.4. From Lemma 7.1 there exists a subsequence $\left\{u_{h^{\prime}}\right\}_{h^{\prime}>0}$ converging in $L^{\infty}\left(J ; L^{1}(\Omega)\right)$ to a limit $u$. Now, by Lemma 7.5 , we have

$$
\lim _{h^{\prime} \rightarrow 0} \Theta\left(u_{h^{\prime}}, c ; V_{h^{\prime}} ; \varphi\right) \leq 0,
$$


for every $c \in \mathbb{R}$ and nonnegative $\varphi \in \mathcal{C}^{1}\left(\bar{Q}_{T}\right)$. Thus, by Lemma 7.2, we see that

$$
\lim _{h^{\prime} \rightarrow 0} \Theta\left(u_{h^{\prime}}, c ; V_{h^{\prime}} ; \varphi\right)=\Theta(u, c ; V ; \varphi) \leq 0 .
$$

This implies that $u$ is the unique solution of (3.10). Consequently, the whole sequence $\left\{u_{h}\right\}_{h>0}$ converges to $u$, and thus Theorem 3.4 is proven.

8. Numerical results. This section reports on numerical results with the finite element method (2.2) for three problems. They are designed to show the performance of the method and to indicate the convergence properties. In all examples the CFL condition (3.6) is required to hold.

FIG. 1. The '- ' , '- - ', and '...' represent the exact $u$ and approximate solution $u_{h}$ with $h=.001$ and $h=.01$.

Example 1. In this example we consider a convecting Gaussian hill in one space dimension. Specifically, we solve equation (1.1) with $\Phi=1, V=10, D=0.1$, and $K=0$ on the interval $[0,6]$. The initial datum $u_{\text {init }}$ is given by

$$
u_{\text {init }}(x)=\mathrm{e}^{-\pi x^{2}} \text {. }
$$

As a pure initial-value problem, this leads to the analytical solution

$$
u_{a}(t, x)=\frac{1}{\sqrt{1+4 \pi D t}} \mathrm{e}^{-\frac{\pi(x-V t)^{2}}{1+4 \pi D t}}
$$

We obtain an initial-boundary-value problem with the same solution by imposing the 
Dirichlet boundary condition:

$$
u(t, 0)=u_{a}(t, 0), \quad u(t, 6)=u_{a}(t, 6)
$$

In Figure 1 we display the analytical solution $u_{a}$ and the approximate solution $u_{h}$ at time $T=0.25$. In Table 1 we display the errors and their respective order of convergence at the same time. From the table we see that the scheme is first-order accurate both in $L^{1}$ and in $L^{\infty}$ for the concentration. This shows that the scheme (2.2) is first-order accurate in both spaces when the solution of the differential equation is smooth. Also, Figure 1 agrees with the stability property given in Theorem 3.1. Finally, our numerical experiments report (not shown here) that, if the CFL condition (3.2) is violated, then the stability result (3.5) and the TVB boundedness (3.7) are no longer valid.

\begin{tabular}{|c|l|l|l|l|}
\hline $1 / \Delta x$ & $L^{\infty}$-error $\left(\times 10^{2}\right)$ & $L^{\infty}$-order & $L^{1}$-error $\left(\times 10^{2}\right)$ & $L^{1}$-order \\
\hline 50 & 6.09 & - & 5.10 & - \\
\hline 100 & 3.57 & 0.76 & 2.50 & 1.03 \\
\hline 200 & 1.89 & 0.91 & 1.20 & 0.99 \\
\hline 400 & 0.99 & 0.93 & 0.62 & 1.02 \\
\hline 800 & 0.51 & 0.98 & 0.29 & 1.09 \\
\hline
\end{tabular}

Table 1. Convergence of $u_{h}$ in $(0,6)$ at $T=.25$.

Example 2. In this example we consider a problem whose solution displays a discontinuity. The problem has the data: $\Phi=1, V=-0.5$, and $K=0$. The boundary and initial conditions are given by

$$
\begin{aligned}
& u(t, 0)=0, \quad u(t, 1)=1, \quad t>0 \\
& u_{\text {init }}(x, 0)=0, \quad x \in[0,1]
\end{aligned}
$$

The exact and approximate 'nonviscous' solution (i.e., in the case of $D=0$ ) and the 'viscous' solution with $D=10^{-3}$ at $T=0.5$ are displayed in Figure 2 . Notice that the biggest error in the approximation of $u$ occurs around the location of the discontinuity $x=0.75$. In Table 2 we show the errors and their convergence orders with $D=0$. Note that the orders of convergence in $L^{1}$ and $L^{\infty}$ are nearly $1 / 2$. This implies that the presence of discontinuity has an effect on the convergence. Finally, from Figure 2, we see that the 'nonviscous' solution is quite close to the 'viscous' solution. 


\begin{tabular}{|c|l|l|l|l|}
\hline $1 / \Delta x$ & $L^{\infty}$-error $\left(\times 10^{2}\right)$ & $L^{\infty}$-order & $L^{1}$-error $\left(\times 10^{2}\right)$ & $L^{1}$-order \\
\hline 50 & 8.65 & - & 9.75 & - \\
\hline 100 & 6.15 & 0.48 & 8.09 & 0.26 \\
\hline 200 & 5.02 & 0.29 & 6.32 & 0.37 \\
\hline 400 & 3.76 & 0.43 & 4.36 & 0.54 \\
\hline 800 & 2.49 & 0.60 & 2.77 & 0.65 \\
\hline
\end{tabular}

Table 2. Convergence of $u_{h}$ in $(0,1)$ at $T=.5$.

FIG. 2. The '—' , '...', and '- - ' represent $u$ with $D=0$ and the approximate solution $u_{h}$ with $D=.0001$ and $D=0$.

Example 3. In the third example we consider a two-dimensional problem which has a shock. The data are set as follows: $\Omega=(0,1)^{2}, \Phi=1, V=\left(\cos \left(\frac{8}{\pi}\right), \sin \left(\frac{8}{\pi}\right)\right)$, 
$K=0$, and $D=10^{-3}$. The Neumann and Dirichlet boundaries $\partial \Omega_{1}$ and $\partial \Omega_{2}$ are defined by

$$
\begin{aligned}
& \partial \Omega_{1}=\{(x, y): 0 \leq x \leq 1, y=1\} \\
& \partial \Omega_{2}=\partial \Omega \backslash \partial \Omega_{1},
\end{aligned}
$$

and the boundary and initial data by

$$
\begin{aligned}
u_{D} & = \begin{cases}1, & x=0, \frac{1}{2}<y<1, \\
0, & \text { elsewhere }\end{cases} \\
u_{\text {init }} & = \begin{cases}1, & 0 \leq x \leq 1, \frac{1}{2}<y \leq 1, \\
0, & \text { elsewhere }\end{cases}
\end{aligned}
$$

The approximate solution of this problem obtained using the method (2.2) with $\Delta x=$ $\Delta y=10^{-2}$ at time $T=2$ is shown in Figure 3 . The graph clearly shows that the method can capture the shock around the location $y=1 / 2$.

FIG. 3. The approximate solution $u_{h}$ on $(0,1)^{2}$.

Example 4. In the final example we test the sharpness of the bounds appearing in (3.7) and (3.8) when $D \neq 0$ and $\left\|u_{D}\right\|_{L^{\infty}\left(J ; B V\left(\partial \Omega_{2}\right)\right)} \neq 0$. The same set of data are chosen as in Example 3 except that the initial and boundary data are determined by

$$
\begin{array}{ll}
u_{\text {init }}(x, y)=x, & (x, y) \in \Omega, \\
u_{D}(x, y)=x, & (x, y) \in \partial \Omega_{2},
\end{array}
$$


where

$$
\begin{aligned}
& \partial \Omega_{2}=\{(x, y): y=1,0.2<x<0.4\} \cup\{(x, y): y=1,0.6<x<0.8\}, \\
& \partial \Omega_{1}=\partial \Omega \backslash \partial \Omega_{2} .
\end{aligned}
$$

Uniform partitions of $\Omega$ into rectangles are exploited. The TVB bounds on different meshes at $T=1$ are given in Table 3 . From this table we see that the left-hand side of the inequality (3.7) blows up as $h=\Delta x=\Delta y$ converges to zero. Similar results are observed for the bound in (3.8) (not shown here).

\begin{tabular}{|c|l|l|l|l|l|l|}
\hline $1 / h$ & 10 & 20 & 40 & 80 & 160 & 320 \\
\hline TVB & 4.8935 & 9.5977 & 20.451 & 43.507 & 93.602 & 198.30 \\
\hline
\end{tabular}

Table 3 . TVB Bounds of $u_{h}$ in $\Omega$ at $T=1$.

9. A concluding remark. A new finite element method for numerically solving the two-dimensional convection-dominated transport equation in ground water has been formulated and analyzed in this paper. The primary computational advantage of the method is that it is local and thus fully parallelizable, and is conservative. The stability properties of this method and its convergence in a suitable topology have been established. Moreover, the numerical results have shown that the method is first-order accurate when the solution is smooth and is one-half order accurate when the solution has discontinuities, and that the method is non-oscillatory and shockcapturing. Future work will be devoted to obtaining error estimates for both cases of zero and nonzero coefficient $D$.

Acknowledgments. The authors wish to thank the referees for their comments leading to an improved presentation of this paper.

\section{REFERENCES}

[1] M. B. Allen iti, G. A. Behie, and J. A. Trangenstein, Multiphase Flow in Porous Media, Lecture Notes in Engineering, Springer-Verlag, 1988.

[2] J. W. BarRett AND K. W. Morton, Approximate symmetrization and Petrov-Galerkin methods for diffusion-convection problems, Comp. Meth. Appl. Mech. Engng., 45 (1984), pp. 97122.

[3] Zhangxin Chen, Finite element analysis of the $1 D$ full drift diffusion semiconductor model, SIAM J. Numer. Anal, 32 (1995), pp. 455-483.

[4] ZhangXin Chen, Numerical simulation of multiphase flow in groundwater hydrology, in Finite Element Modeling of Environmental Problems, Surface and Subsurface Flow and Transport, G. Carey, ed., John Wiley \& Sons, 1995, pp. 361-374.

[5] Zhangin Chen and B. Cockburn, Error estimates for a finite element method for the driftdiffusion semiconductor device equations, SIAM J. Numer. Anal., 31, pp. 1062-1089.

[6] Zhangxin Chen and B.Cockburn, Convergence of a finite element method for the driftdiffusion semiconductor device equations: the multidimensional case, Numer. Math., 71 (1995), pp. 1-28.

[7] Zhangxin Chen and J. Douglas, JR., Approximation of coefficients in hybrid and mixed methods for nonlinear parabolic problems, Mat. Aplic. Comp., 10 (1991), pp. 137-160.

[8] Zhangxin Chen, Bernardo Cockburn, Joseph W. Jerome and Chiwang Shu, Mixed$R K D G$ finite element methods for the 2-D hydrodynamic model for semiconductor device 
simulation, VLSI Designs, 3 (1995), pp. 1-14.

[9] I. Christie, D. F. Griffiths, and A. R. Mitchell, Finite element methods for second order differential equations with significant first derivatives, Int. J. Num. Engrg., 10 (1976), pp. 1389-1396.

[10] B. CockBurn and I. TRiandaf, Convergence of a finite element method for the drift diffusion semiconductor device equations, Math. Comp., 59 (1992), pp. 383-401.

[11] B. CockBurn And I. TRIANDAF, Error estimates for a finite element method for the drift diffusion semiconductor device equations: the zero-diffusion case, Math. Comp., 63, pp. 5176.

[12] B. Cockburn and C.W. Shu, TVB Runge-Kutta local projection discontinuous Galerkin finite element method for scalar conservation laws II: general framework, Math. Comp., 52 (1989), pp. 411-435.

[13] M. CRAndall And A. MAJdA, Monotone difference approximations for scalar conservation laws, Math. Comp., 34 (1980), pp. 1-21.

[14] J. Douglas, JR. And J. Roberts, Global estimates for mixed methods for second order elliptic problems, Math. Comp., 45 (1985), pp. 39-52.

[15] J. Douglas, JR. And T. F. Russell, Numerical methods for convection-dominated diffusion problems based on combining the method of characteristics with finite element or finite difference procedures, SIAM J. Num. Anal., 19 (1982), pp. 871-885.

[16] R. E. EwIng, ED., Research Frontiers in Applied Mathematics, Vol. 1, SIAM, Philadelphia, 1983.

[17] R. E. Ewing And T. F. Russell, Multistep Galerkin methods along characteristics for convectiondiffusion problems, Advances in Computer Methods for PDEs IV, Vichnevetsky and Stepleman, (eds.) IMACS, Rutgers Univ. (1981), pp. 28-36.

[18] R. E. Ewing, T. F. Russell, And M. F. Wheeler, Convergence analysis of an approximation of miscible displacement in porous media by mixed finite elements and a modified method of characteristics, Comp. Meth. Appl. Mech. Engng., 47 (1984), pp. 73-92.

[19] R. D. Lazarov, I. D. Mishev, and P. S. Vassilevski, Finite volume methods with local refinement for convection-diffusion problems, Technical Report 92-50, Department of Mathematics, UCLA, Los Angeles, 1992.

[20] S. McCormick, Multilevel Adaptive Methods for Partial Differential Equations, SIAM, Philadelphia, 1989.

[21] P.A. Raviart and J.M. Thomas, A mixed finite element method for second order elliptic problems, Lecture Notes in Mathematics, Springer-Verlag, 1977.

[22] T. Russell and M. Wheeler, Finite element and finite difference methods for continuous flows in porous media, in Chapter II, The Mathematics of Reservoir Simulation, R. Ewing, ed., Frontiers in Applied Mathematics 1, Society for Industrial and Applied Mathematics, Philadelphia, 1983, pp. 35-106. 